\title{
ON THE NUMBER OF RATIONAL POINTS ON DRINFELD MODULAR VARIETIES OVER FINITE FIELDS
}

\author{
MIHRAN PAPIKIAN
}

\begin{abstract}
Drinfeld and Vladut proved that Drinfeld modular curves have

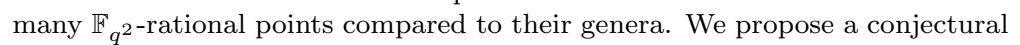
generalization of this result to higher dimensional Drinfeld modular varieties, and prove a theorem giving some evidence for the conjecture.
\end{abstract}

\section{IntROduCtion}

Let $q$ be a power of a prime $p$ and let $\mathbb{F}_{q}$ denote the finite field with $q$ elements. Let $X$ be a smooth, geometrically connected, $d$-dimensional variety defined over $\mathbb{F}_{q}$. Fix an algebraic closure $\overline{\mathbb{F}}_{q}$ of $\mathbb{F}_{q}$. Also, fix a prime number $\ell \neq p$ and an algebraic closure $\overline{\mathbb{Q}}_{\ell}$ of the field $\mathbb{Q}_{\ell}$ of $\ell$-adic numbers. Grothendieck's theory of étale cohomology produces the $\ell$-adic cohomology groups with compact supports

$$
H^{*}(X):=H_{c}^{*}\left(X \otimes_{\mathbb{F}_{q}} \overline{\mathbb{F}}_{q}, \overline{\mathbb{Q}}_{\ell}\right) .
$$

These groups are finite dimensional $\overline{\mathbb{Q}}_{\ell}$-vector spaces endowed with an action of the Galois group $\operatorname{Gal}\left(\overline{\mathbb{F}}_{q} / \mathbb{F}_{q}\right)$. It is known that $H^{i}(X)=0$ for $i>2 d$, cf. [20, Ch. VI]. Denote by $h^{i}(X):=\operatorname{dim}_{\overline{\mathbb{Q}}_{\ell}} H^{i}(X)$ the (compact) $\ell$-adic Betti numbers of $X$.

Let $\operatorname{Frob}_{q}$ be the inverse of the standard topological generator $x \mapsto x^{q}$ of $\operatorname{Gal}\left(\overline{\mathbb{F}}_{q} / \mathbb{F}_{q}\right)$, i.e., the so-called geometric Frobenius element. Assume $H^{i}(X) \neq$ 0 . Denote the eigenvalues of $\operatorname{Frob}_{q}$ acting on $H^{i}(X)$ by $\alpha_{i, 1}, \alpha_{i, 2}, \ldots, \alpha_{i, s}$ (here $\left.s=h^{i}(X)\right)$. Deligne proved that $\left\{\alpha_{i, j}\right\}$ are algebraic numbers. Moreover, for any isomorphism $\iota: \overline{\mathbb{Q}}_{\ell} \rightarrow \mathbb{C}$ the absolute value $\left|\iota\left(\alpha_{i, j}\right)\right|$ is independent of $\iota$ and is equal to $q^{m / 2}$ for some $0 \leq m \leq i$; see [3, Thm. 3.3.1].

For an integer $n \geq 1$ denote by $\mathbb{F}_{q^{n}}$ the degree $n$ extension of $\mathbb{F}_{q}$, and let $X\left(\mathbb{F}_{q^{n}}\right)$ be the set of $\mathbb{F}_{q^{n}}$-rational points on $X$. By the Grothendieck-Lefschetz trace formula [20, Thm. 13.1]

$$
\# X\left(\mathbb{F}_{q^{n}}\right)=\sum_{i \geq 0}(-1)^{i} \operatorname{Tr}\left(\operatorname{Frob}_{q}^{n} \mid H^{i}(X)\right)=\sum_{i \geq 0}(-1)^{i} \sum_{j=1}^{h^{i}(X)} \alpha_{i, j}^{n} .
$$

If one combines this formula with Deligne's bounds, then there results the estimate

$$
\# X\left(\mathbb{F}_{q^{n}}\right) \leq \sum_{i \geq 0} q^{i n / 2} h^{i}(X)
$$

When $X$ is a curve, this estimate is equivalent to Weil's famous bound.

Since the early 80's, partly due to Goppa's construction of algebra-geometric codes, the question of the "optimality" of the bound (1.1) received a considerable

Key words and phrases. Drinfeld modular varieties, rational points, Drinfeld-Vladut bound. Date: January 31, 2006. 
amount of attention. More precisely, it became important to know whether there exist varieties over $\mathbb{F}_{q}$ which have many rational points compared to their Betti numbers. One way to formulate this problem is as follows: Assume $d, q$ and $n$ are fixed. For a smooth, geometrically connected $d$-dimensional variety $X$ over $\mathbb{F}_{q}$ put $h(X):=\sum_{i} h^{i}(X)$. How large can the ratio $\# X\left(\mathbb{F}_{q^{n}}\right) / h(X)$ be when $h(X) \gg q$ ? Not much is known about this question beyond dimension 1.

We recall the principal results for the case of curves, i.e., for $d=1$. Refining an idea of Ihara, Drinfeld and Vladut [26] proved that when $h(X) \gg q$

$$
\frac{X\left(\mathbb{F}_{q^{n}}\right)}{h(X)} \leq \frac{q^{n / 2}-1}{2}
$$

(Note that Weil's bound only gives $X\left(\mathbb{F}_{q^{n}}\right) / h(X) \leq q^{n / 2}$.) Now the modular curves (classical, Shimura, Drinfeld) enter the picture in a key manner, since they provide examples of curves which attain the previous bound for $n=2$ (and in fact the modular curves are the only known such examples). We recall the result for the Drinfeld modular curves, which is due to Vladut [19]. First we need to introduce some notation.

Let $T$ be a transcendental parameter over $\mathbb{F}_{q}$, and let $A=\mathbb{F}_{q}[T]$ be the ring of polynomials in $T$ with $\mathbb{F}_{q}$ coefficients. Let $\mathfrak{n} \triangleleft A$ be an ideal, and let $M_{\mathfrak{n}}^{d+1}$ be the Drinfeld modular scheme parametrizing Drinfeld $A$-modules of rank $(d+1)$ with full level $\mathfrak{n}$ structure (we refer to $\S 4$ for the definitions). Drinfeld proved that $M_{\mathfrak{n}}^{d+1} \rightarrow \operatorname{Spec}\left(A\left[\mathfrak{n}^{-1}\right]\right)$ is a smooth affine scheme of pure relative dimension $d$. Its fibre over a prime $\mathfrak{l} \triangleleft A\left[\mathfrak{n}^{-1}\right]$ will be denoted by $M_{\mathfrak{n}, \mathfrak{l}}^{d+1}$. The group $\operatorname{GL}_{d+1}(A / \mathfrak{n})$ acts on $M_{\mathfrak{n}, \mathfrak{l}}^{d+1}$. Denote by $X_{\mathfrak{n}, \mathfrak{l}}^{d+1}$ the quotient of $M_{\mathfrak{n}, \mathfrak{l}}^{d+1}$ under the action of $(A / \mathfrak{n})^{\times}$ embedded into $\mathrm{GL}_{d+1}(A / \mathfrak{n})$ as the subgroup of scalar matrices.

Assume $\mathfrak{n}=\mathfrak{p} \neq(T)$ is a prime of odd degree. In Chapter II of [19] Vladut shows that $X_{\mathfrak{p}, T}^{2}$ is a smooth, affine, geometrically connected curve defined over $\mathbb{F}_{q}$, $h\left(X_{\mathfrak{p}, T}^{2}\right) \rightarrow \infty$ when $\operatorname{deg}(\mathfrak{p}) \rightarrow \infty$, and

$$
\liminf _{\operatorname{deg}(\mathfrak{p}) \rightarrow \infty}\left(\frac{\# X_{\mathfrak{p}, T}^{2}\left(\mathbb{F}_{q^{2}}\right)}{h\left(X_{\mathfrak{p}, T}^{2}\right)}\right) \geq \frac{q-1}{2} .
$$

Therefore, by comparing with (1.2), we have

$$
\lim _{\operatorname{deg}(\mathfrak{p}) \rightarrow \infty}\left(\frac{\# X_{\mathfrak{p}, T}^{2}\left(\mathbb{F}_{q^{2}}\right)}{h\left(X_{\mathfrak{p}, T}^{2}\right)}\right)=\frac{q-1}{2} .
$$

This result can be extended to other Drinfeld modular curves having different types of level structures, and also to their canonical compactifications; see [19] or [10].

Almost nothing is known about the accumulation points of the set of rational numbers $S(q, n, d):=\left\{\# X\left(\mathbb{F}_{q^{n}}\right) / h(X) \mid \operatorname{dim}(X)=d\right\} \subset\left[0, q^{d n}\right]$ unless $d=1$. Even in the case of curves there are still some fundamental open problems. For example, the largest accumulation point of $S(p, n, 1)$ is not known for any $p$ unless $n$ is even, in which case the answer is $\left(p^{n / 2}-1\right) / 2$.

In this paper we would like to propose a conjectural generalization of the result of Vladut and Drinfeld to an arbitrary $d \geq 1$. Fix $q$ and $d$, and let $n=d+1$.

Definition 1.1. Let $\mathfrak{p} \triangleleft A$ be a prime. We say that $\mathfrak{p}$ is admissible if $x \mapsto x^{n}$ is an automorphism of $(A / \mathfrak{p})^{\times} / \mathbb{F}_{q}^{\times}$. 
It is easy to show that there are infinitely many admissible primes; see Lemma 4.6. (Note that the primes of odd degree are admissible when $d=1$.) In $\S 4$ we will prove that for an admissible prime $\mathfrak{p} \neq(T), X_{\mathfrak{p}, T}^{n}$ is a smooth, affine, geometrically connected variety of dimension $d$ defined over $\mathbb{F}_{q}$. Moreover, $h\left(X_{\mathfrak{p}, T}^{n}\right) \rightarrow \infty$ when $\operatorname{deg}(\mathfrak{p}) \rightarrow \infty$.

\section{Conjecture 1.2.}

$$
\lim _{\operatorname{deg}(\mathfrak{p}) \rightarrow \infty}\left(\frac{\# X_{\mathfrak{p}, T}^{n}\left(\mathbb{F}_{q^{n}}\right)}{h\left(X_{\mathfrak{p}, T}^{n}\right)}\right)=\frac{1}{n} \prod_{i=1}^{n-1}\left(q^{i}-1\right),
$$

where the limit is over the admissible primes not equal to $(T)$.

When $n=2$, this is exactly (1.3). From a general perspective, the conjecture specifies an accumulation point of $S(q, n, n)$.

Let $F$ be the fraction field of $A$. Fix a separable closure $\bar{F}$ of $F$. Denote by $\eta: A\left[\mathfrak{n}^{-1}\right] \hookrightarrow F$ the generic point of $\operatorname{Spec}\left(A\left[\mathfrak{n}^{-1}\right]\right)$ and by $M_{\eta}^{n}(\mathfrak{n}):=M^{n}(\mathfrak{n}) \otimes_{A[\mathfrak{n}-1]} F$ the generic fibre of $M^{n}(\mathfrak{n})$. Consider the virtual $\operatorname{Gal}(\bar{F} / F)$-module

$$
\mathcal{H}=\sum_{i \geq 0}(-1)^{i} H_{c}^{i}\left(M_{\eta}^{n}(\mathfrak{n}) \otimes_{F} \bar{F}, \overline{\mathbb{Q}}_{\ell}\right) .
$$

Write $\mathcal{H}$ as a sum of irreducible modules with integral coefficients $\mathcal{H}=\sum_{j \geq 0} a_{j} \mathcal{H}_{j}$. Assume

$$
\sum_{i \geq 0} \operatorname{dim}_{\overline{\mathbb{Q}}_{\ell}} H_{c}^{i}\left(M_{\eta}^{n}(\mathfrak{n}) \otimes_{F} \bar{F}, \overline{\mathbb{Q}}_{\ell}\right) \sim \sum_{j \geq 0}\left|a_{j}\right| \operatorname{dim}_{\overline{\mathbb{Q}}_{\ell}} \mathcal{H}_{j}
$$

where $\sim$ means that the left-hand side divided by the right-hand side tends to 1 as $\operatorname{deg}(\mathfrak{n}) \rightarrow \infty$. The assumption essentially says that the same irreducible representation of $\operatorname{Gal}(\bar{F} / F)$ tends to appear only in the cohomology groups of the same parity. Most likely this is always true, and will follow from a certain refinement of the Langlands conjecture over function fields; we will say more about this in $\S 4$. In any case, (1.4) is true for $n=2$ as easily follows from Drinfeld's theorem [4]. The main result of this paper is the following evidence for Conjecture 1.2:

Theorem 1.3. Under the assumption (1.4) we have

$$
\begin{gathered}
\frac{1}{n} \prod_{i=1}^{n-1}\left(q^{i}-1\right) \leq \liminf _{\operatorname{deg}(\mathfrak{p}) \rightarrow \infty}\left(\frac{\# X_{\mathfrak{p}, T}^{n}\left(\mathbb{F}_{q^{n}}\right)}{h\left(X_{\mathfrak{p}, T}^{n}\right)}\right), \\
\quad \limsup _{\operatorname{deg}(\mathfrak{p}) \rightarrow \infty}\left(\frac{\# X_{\mathfrak{p}, T}^{n}\left(\mathbb{F}_{q^{n}}\right)}{h\left(X_{\mathfrak{p}, T}^{n}\right)}\right) \leq q^{\frac{n(n-1)}{2}} .
\end{gathered}
$$

Note that the degrees of the upper and lower bounds in the theorem, as polynomials in $q$, are the same, so the bounds are not that far from each other. Moreover, we will show that the upper bound is exactly the $\operatorname{limit} \lim _{\mathfrak{p}}\left(\sum_{i, j}\left|\alpha_{i, j}^{n}\right|\right) / h\left(X_{\mathfrak{p}, T}^{n}\right)$, so the Drinfeld modular varieties $X_{\mathfrak{p}, T}^{n}$ come close to having as many $\mathbb{F}_{q^{n} \text {-rational }}$ points as the Weil-Deligne bound allows.

The organization of the paper and the outline of the proof of Theorem 1.3 are as follows: The definition and the main properties of Drinfeld modular varieties $M^{n}(\mathfrak{n})$ are recalled in $\S 4$. In the same section we show that $X_{\mathfrak{p}, T}^{n}$ are geometrically 
irreducible when $\mathfrak{p}$ is admissible. The proof relies on the analogue of the Weil pairing for Drinfeld modules constructed by van der Heiden. To get an estimate

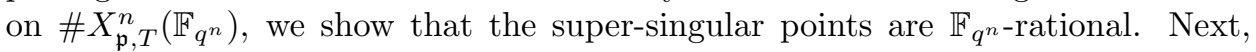
under the assumption (1.4), we use Laumon's proof of a special case of Langlands conjecture over function fields to reduce the calculation of the asymptotic size of $h\left(X_{\mathfrak{p}, T}^{n}\right)$ to the calculation of the dimension of a certain space of cusp forms on $\mathrm{GL}_{n}$. A theorem of Harder relates the dimension of this space of cusp forms to the Euler-Poincaré characteristic of the quotient of the Bruhat-Tits building of $\mathrm{PGL}_{n}$ under the action of level-n principal congruence subgroup of $\mathrm{GL}_{n}(A)$; see $\S 3.5$. The calculation of the Euler-Poincaré characteristic is carried out in $\S 3$. Our methods are combinatorial. The final result expresses the Euler-Poincaré characteristic as a sum of the special values of a partial zeta-function of $F$. This can be interpreted as a Gauss-Bonnet type formula in the non-archimedean setting, and is of independent interest. Once we know the asymptotic size of $h\left(X_{\mathfrak{p}, T}^{n}\right)$ and a lower bound on $\# X_{\mathfrak{p}, T}^{n}\left(\mathbb{F}_{q^{n}}\right)$, the lower bound in Theorem 1.3 easily follows. To get the upper bound we use the Ramanujan-Petersson conjecture proven in this setting by Laumon [18].

Acknowledgements. The work on this article was started while I enjoyed the hospitality of IHÉS. The financial support of Institut Post-Doctoral Européen is gratefully acknowledged. I thank G. Andrews and A. Yang for helpful communications related to the proof of Lemma 3.2.

\section{Conventions}

The purpose of this section is to introduce the terminology and notation which will be used in later sections of the paper.

2.1. Simplicial complexes. Recall that an $n$-dimensional simplex $s$ (or an $n$ simplex, for short) is the smallest convex set in a real vector space containing $n+1$ points $v_{0}, v_{1}, \ldots, v_{n}$ in general position. The points $v_{i}$ are the vertices of the simplex $s$. A specific ordering of the vertices of $s$ is called an orientation of $s$; two orientations which can reach each other through an even number of permutations of $v_{i}$ 's are regarded as equal. Hence, every positive dimensional simplex has exactly two orientations. Any simplex spanned by a subset of $\left\{v_{0}, v_{1}, \ldots, v_{n}\right\}$ is called a face of $s$. We say that $\sigma$ is an oriented face of $s$, and write it as $s>\sigma$, if $\sigma$ is a face of $s$ and the orientation of $\sigma$ is the restriction of that of $s$.

A simplicial complex $\mathcal{D}$ is a collection of simplices such that a face of a simplex of $\mathcal{D}$ is in $\mathcal{D}$, and the intersection of two simplices of $\mathcal{D}$ is a face of each of them. The dimension of $\mathcal{D}$ is the supremum of the dimensions of its simplices. A subcollection $\mathcal{D}^{\prime}$ of $\mathcal{D}$ that contains all the faces of its elements is called a subcomplex of $\mathcal{D}$.

A $\Delta$-complex, as defined in [16], is a quotient space of a collection of disjoint simplices obtained by identifying certain of their faces via canonical linear homeomorphisms that preserve the ordering of vertices. From the point of view of homology theory, $\Delta$-complexes are equivalent to simplicial complexes. In fact, it is easy to see that a simplicial complex is a $\Delta$-complex, and a $\Delta$-complex is homeomorphic to a simplicial complex. (Note also that simplicial complexes are the $\Delta$-complexes whose simplices are uniquely determined by their vertices.) Denote the set of oriented $i$-simplicies of a $\Delta$-complex $\mathcal{D}$ by $S_{i}(\mathcal{D})$, and the set of nonoriented $i$-simplices by $\widetilde{S}_{i}(\mathcal{D})$. We also denote the set of vertices of $\mathcal{D}$ by $\operatorname{Ver}(\mathcal{D})$, so $\operatorname{Ver}(\mathcal{D})=S_{0}(\mathcal{D})=\widetilde{S}_{0}(\mathcal{D})$ 
The homology groups $H_{*}(\mathcal{D}, R)$ (and the cohomology groups $H^{*}(\mathcal{D}, R)$ ) of a $\Delta$ complex $\mathcal{D}$ with coefficients in a ring $R$ are defined in a usual manner; see $[16, \mathrm{Ch}$. 2]. We simply write $H_{*}(\mathcal{D})$ for $H_{*}(\mathcal{D}, \mathbb{Q})$. Assume $\mathcal{D}$ is $n$-dimensional, and $H_{i}(\mathcal{D})$ are finite dimensional. The Euler-Poincaré characteristic of $\mathcal{D}$ is

$$
\chi(\mathcal{D}):=\sum_{i=0}^{n}(-1)^{i} \operatorname{dim}_{\mathbb{Q}} H_{i}(\mathcal{D}) .
$$

If $\mathcal{D}$ is finite, then, as is easy to check,

$$
\chi(\mathcal{D})=\sum_{i=0}^{n}(-1)^{i} \# \widetilde{S}_{i}(\mathcal{D}) .
$$

Let $G$ be a group acting on the vertices of $\mathcal{D}$. We say that $G$ preserves the simplicial structure of $\mathcal{D}$, or simply, $G$ acts on $\mathcal{D}$, if for any $n$-simplex $\left\{v_{0}, \ldots, v_{n}\right\}$ of $\mathcal{D}$ and any $g \in G$ the set $\left\{g v_{0}, \ldots, g v_{n}\right\}$ is also a $n$-simplex of $\mathcal{D}$.

If $G$ acts on $\mathcal{D}$ then we can construct a $\Delta$-complex $\mathcal{D} / G$, which is naturally the quotient space of this action. For $s \in S_{i}(\mathcal{D})$ denote by $G s$ the orbit of the action of $G$ on $s$. Let $S_{i}(\mathcal{D} / G)=\left\{G s \mid s \in S_{i}(\mathcal{D})\right\}$ be the set of such orbits. Set $G \sigma<G s$ if and only if there is $\sigma^{\prime}<s$ such that $G \sigma^{\prime}=G \sigma$. By gluing the simplices in $S_{i}(\mathcal{D} / G)$ and $S_{j}(\mathcal{D} / G)$ for $0 \leq i, j \leq \operatorname{dim}(\mathcal{D})$, along their common faces, we obtain the desired $\Delta$-complex. Note that $\operatorname{dim}(\mathcal{D} / G)=\operatorname{dim}(\mathcal{D})$.

If $G$ acts on $\mathcal{D}$ then for any oriented simplex $w$ of $\mathcal{D}$ we denote by $\operatorname{Stab}_{G}(w)$ or $G_{w}$ the stabilizer of $w$ in $G$.

(The $\Delta$-complexes which arise in this paper turn out to be simplicial; see Remark 3.16. This extra property will not play a significant role in what follows, as we are primarily interested in the homology of these complexes.)

2.2. Levi decomposition. Let $n$ be a positive integer. An ordered partition of $n$ is an expression of $n$ as an ordered sum of positive integers. We will write ordered partitions as row vectors. Let $\mathbf{P}(n)$ be the set of all ordered partitions of $n$, so $\mathbf{p}=\left(p_{1}, \ldots, p_{h}\right) \in \mathbf{P}(n)$ if $n=p_{1}+\cdots+p_{h}$, and all $p_{i} \in \mathbb{Z}_{>0}$. It is easy to check that $\mathbf{P}(n)$ has $2^{n-1}$ elements. Define the length of $\mathbf{p}=\left(p_{1}, \ldots, p_{h}\right)$ to be $\ell(\mathbf{p})=h$.

To each $\mathbf{p}=\left(p_{1}, \ldots, p_{h}\right) \in \mathbf{P}(n)$ we associate the subgroup $P_{\mathbf{p}}$ of $\mathrm{GL}_{n}$ consisting of matrices of the form

$$
\left(\begin{array}{cccc}
G_{11} & G_{12} & \cdots & G_{1 h} \\
0 & G_{22} & \cdots & G_{2 h} \\
\vdots & & \ddots & \vdots \\
0 & 0 & \cdots & G_{h h}
\end{array}\right)
$$

where $G_{i j}$ is a $p_{i} \times p_{j}$ block. The group $P_{\mathbf{p}}$ is a semidirect product

$$
P_{\mathbf{p}}=M_{\mathbf{p}} \rtimes U_{\mathbf{p}},
$$

where $M_{\mathbf{p}}$ is characterized by the condition that $G_{i j}=0$ unless $i=j$, and the normal subgroup $U_{\mathbf{p}}$ is characterized by the condition that each $G_{i i}$ is the identity matrix in $\mathrm{GL}_{p_{i}}$. The groups $P_{\mathbf{p}}$ are called the standard parabolic subgroups of $\mathrm{GL}_{n}$. The subgroup $U_{\mathbf{p}}$ is called the unipotent radical of $P_{\mathbf{p}}$, and $M_{\mathbf{p}}$ is called the standard Levi subgroup of $P_{\mathbf{p}}$. Evidently,

$$
M_{\mathbf{p}} \cong \mathrm{GL}_{p_{1}} \times \cdots \times \mathrm{GL}_{p_{h}} .
$$

The decomposition $P_{\mathbf{p}}=M_{\mathbf{p}} \rtimes U_{\mathbf{p}}$ is called the Levi decomposition of $P_{\mathbf{p}}$. 
Let $\Lambda$ be the set $\{2,3, \ldots, n\}$. To each subset $I \subseteq \Lambda$ we associate an ordered partition $\mathbf{p}(I) \in \mathbf{P}(n)$ as follows. First, put $\mathbf{p}(\Lambda)=(n)$. If $I \varsubsetneqq \Lambda$, let

$$
\Lambda-I=\left\{i_{1}<i_{2}<\cdots<i_{k}\right\} .
$$

Now let $\mathbf{p}(I)=\left(i_{1}-1, i_{2}-i_{1}, \ldots, i_{k}-i_{k-1}, n+1-i_{k}\right)$. Note that $\mathbf{p}(\emptyset)=(1,1, \ldots, 1)$. It is easy to see that $I \rightarrow \mathbf{p}(I)$ is a one-to-one correspondence between the subsets of $\Lambda$ and the elements of $\mathbf{P}(n)$. Denote by $P_{I}, M_{I}, U_{I}$ the groups $P_{\mathbf{p}(I)}, M_{\mathbf{p}(I)}, U_{\mathbf{p}(I)}$, respectively.

2.3. Notation. From now on, unless specified otherwise, the following notation is fixed:

$n \geq 2$ is a fixed integer;

$G=\mathrm{GL}_{n}$;

$B$ is the Borel subgroup of upper-triangular matrices of $G$;

$Z$ is the center of $G$;

$\mathbb{F}_{q}$ is the finite field of $q$ elements, where $q$ is a power of the prime $p$; $A=\mathbb{F}_{q}[T]$ is the ring of polynomials in $T$ with coefficients in $\mathbb{F}_{q}$;

$F=\mathbb{F}_{q}(T)$ is the fraction field of $A$ (equiv. the field of rational functions on $\mathbb{P}_{\mathbb{F}_{q}}^{1}$ );

$F_{v}$ is the completion of $F$ at the place $v$;

$\pi_{v}$ is a uniformizer of $F_{v}$;

$\operatorname{ord}_{v}$ is the canonical valuation on $F_{v}$ normalized by $\operatorname{ord}_{v}\left(\pi_{v}\right)=1$;

$\mathcal{O}_{v}=\left\{x \in F_{v} \mid \operatorname{ord}_{v}(x) \geq 0\right\}$ is the ring of integers in $F_{v}$;

$\mathfrak{p}_{v}=\pi_{v} \mathcal{O}_{v}$ is the maximal ideal of $\mathcal{O}_{v}$;

$\mathbb{F}_{v}$ is the residue field $\mathcal{O}_{v} / \mathfrak{p}_{v}$

$q_{v}$ is the order of the finite field $\mathbb{F}_{v}$;

$\mathbb{A}$ is the ring of adeles of $F$;

$\mathbb{A}^{\times}$is the group of ideles of $F$;

$\mathcal{O}=\prod_{v} \mathcal{O}_{v}$

$\mathcal{K}\left(\mathfrak{n}_{v}\right)=\left\{M \in G\left(\mathcal{O}_{v}\right) \mid M \equiv 1 \bmod \mathfrak{n}_{v}\right\}$, where $\mathfrak{n}_{v}$ is an ideal of $\mathcal{O}_{v}$, is the principal congruence subgroup of $G\left(\mathcal{O}_{v}\right)$ of level $\mathfrak{n}_{v}$;

Let $\mathfrak{n}$ be a monic polynomial in $A$. We denote by the same letter the ideal generated by $\mathfrak{n}$ in $A$. If $\mathfrak{p} \triangleleft A$ is a prime ideal, we denote the residue field $A / \mathfrak{p}$ by $\mathbb{F}_{\mathfrak{p}}$. Consider the map $\operatorname{deg}: A \rightarrow \mathbb{Z}$ which to each polynomial $f(T) \in A$ associates its degree in $T$ (by convention, $\operatorname{deg}(0)=\infty$ ). This induces a valuation $w$ on $F$ by $w(a / b)=\operatorname{deg}(b)-\operatorname{deg}(a)$, where $a, b \in A$. The place corresponding to this valuation is denoted by $\infty$. This place will play a special role in what follows. A natural uniformizer at $\infty$ is $1 / T$. Finally, denote

$\Gamma=G(A)$

$\Gamma(\mathfrak{n})=\operatorname{ker}(G(A) \rightarrow G(A / \mathfrak{n}))$;

$\mathbb{A}_{f}=\prod_{v \neq \infty}^{\prime} F_{v}$

$\mathbb{A}_{f}^{\times}=\prod_{v \neq \infty}^{\prime} F_{v}^{\times}$

$\mathcal{O}_{f}=\prod_{v \neq \infty} \mathcal{O}_{v}$.

Note that $\mathbb{A}=\mathbb{A}_{f} \times F_{\infty}, \mathbb{A}=\mathbb{A}_{f}^{\times} \times F_{\infty}^{\times}, \mathcal{O}=\mathcal{O}_{f} \times \mathcal{O}_{\infty}$, and $\mathcal{O}_{f}$ is the completion of $A$ with respect to the ideal topology. 
Let $K$ be a field, and let $V$ be an $n$-dimensional vector space over $K$. A flag in $V$ is a sequence of linear subspaces

$$
\mathcal{F}: 0 \subset V_{1} \subset V_{2} \subset \cdots \subset V_{h-1} \subset V_{h}=V,
$$

where $V_{1} \neq 0$ and $V_{i} \neq V_{i+1}$ for $1 \leq i \leq h-1$. A flag with $h=n$ is called maximal.

\section{Quotients of Bruhat-Tits BuILDing}

In this section we compute the Euler-Poincaré characteristic of the quotient the Bruhat-Tits building of $\mathrm{PGL}_{n}\left(F_{\infty}\right)$ under the action of $\Gamma(\mathfrak{n})$, and relate this number to the dimension of a space of cusp forms on $G(\mathbb{A})$.

3.1. Combinatorial identity. First we prove an identity for $q$-multinomial coefficients, which we will use in $\S 3.4$. Denote

$$
(a)_{k}:=\prod_{i=0}^{k-1}\left(1-a q^{i}\right) \quad \text { and } \quad(a)_{\infty}:=\prod_{i=0}^{\infty}\left(1-a q^{i}\right) .
$$

(For now, $q$ can be thought of just as a fixed parameter.) To each ordered partition $\mathbf{p}=\left(p_{1}, \ldots, p_{h}\right)$ of $m \geq 1$ corresponds the $q$-multinomial coefficient:

$$
\left[\begin{array}{c}
m \\
\mathbf{p}
\end{array}\right]=\frac{(q)_{m}}{(q)_{p_{1}}(q)_{p_{2}} \cdots(q)_{p_{h}}} .
$$

It is well-known that the $q$-multinomial coefficients are polynomials in $q$.

Remark 3.1. Let $\mathbf{p}=\left(p_{1}, \ldots, p_{h}\right) \in \mathbf{P}(m)$. Let $\mathbb{C}\left\langle x_{1}, x_{2}, \ldots, x_{h}\right\rangle$ be the noncommutative polynomial ring where the constants commute with all $x_{i}$ 's and $x_{j} x_{i}=$ $q x_{i} x_{j}$ for any $i<j$. Then $\left[\begin{array}{c}m \\ \mathbf{p}\end{array}\right]$ is the coefficient of $x_{1}^{p_{1}} \cdots x_{h}^{p_{h}}$ in $\left(x_{1}+\cdots+x_{h}\right)^{m}$, which explains the terminology.

\section{Lemma 3.2.}

$$
\sum_{\mathbf{p} \in \mathbf{P}(m)}(-1)^{\ell(\mathbf{p})}\left[\begin{array}{c}
m \\
\mathbf{p}
\end{array}\right]=(-1)^{m} q^{\frac{m(m-1)}{2}} .
$$

Proof. In the proof we will use two formulas of Euler [1, Cor. 2.2]:

$$
1+\sum_{i=1}^{\infty} \frac{x^{i}}{(q)_{i}}=\frac{1}{(x)_{\infty}}
$$

and

$$
1+\sum_{i=1}^{\infty} \frac{(-1)^{i} x^{i} q^{\frac{i(i-1)}{2}}}{(q)_{i}}=(x)_{\infty} .
$$

It is easy to see that the left-hand side of the desired identity is the coefficient of $x^{m} /(q)_{m}$ in

$$
\begin{gathered}
1+\sum_{h=0}^{\infty} \sum_{m=1}^{\infty} \frac{x^{m}}{(q)_{m}}(-1)^{h} \sum_{\substack{p_{1}+\cdots+p_{h}=m \\
p_{1}, \ldots, p_{h} \geq 1}} \frac{(q)_{m}}{(q)_{p_{1}}(q)_{p_{2}} \cdots(q)_{p_{h}}} \\
=1+\sum_{h=1}^{\infty}(-1)^{h} \sum_{p_{1}, \ldots, p_{h} \geq 1} \frac{x^{p_{1}+p_{2} \cdots+p_{h}}}{(q)_{p_{1}}(q)_{p_{2}} \cdots(q)_{p_{h}}} .
\end{gathered}
$$


By (3.1) this last expression is equal to

$$
1+\sum_{h=1}^{\infty}(-1)^{h}\left(\frac{1}{(x)_{\infty}}-1\right)^{h}=\frac{1}{1-\left(1-\frac{1}{(x)_{\infty}}\right)}=(x)_{\infty} .
$$

Now the claim follows from (3.2), as the coefficients of $x^{m} /(q)_{m}$ in the left-hand side of that formula is $(-1)^{m} q^{\frac{m(m-1)}{2}}$.

Remark 3.3. By taking $q \rightarrow 1$ in Lemma 3.2, we get the following identity for the usual multinomial coefficients:

$$
\sum_{\left(p_{1}, \ldots, p_{h}\right) \in \mathbf{P}(m)}(-1)^{h}\left(\begin{array}{c}
m \\
p_{1}, \ldots, p_{h}
\end{array}\right)=(-1)^{m} .
$$

Notation 3.4. For $m \geq 1$, let

$$
\phi(m)=\sum_{\mathbf{p} \in \mathbf{P}(m)}(-1)^{\ell(\mathbf{p})-1}\left(\# P_{\mathbf{p}}\left(\mathbb{F}_{q}\right)\right)^{-1}
$$

Proposition 3.5.

$$
\phi(m)=-\frac{1}{(q)_{m}} .
$$

Proof. Let $g_{k}=\# \mathrm{GL}_{k}\left(\mathbb{F}_{q}\right)$. Then

$$
g_{k}=\prod_{i=0}^{k-1}\left(q^{k}-q^{i}\right)=q^{\frac{k(k-1)}{2}} \prod_{i=1}^{k}\left(q^{i}-1\right)=(-1)^{k} q^{\frac{k(k-1)}{2}}(q)_{k} .
$$

Let $\mathbf{p}=\left(p_{1}, \ldots, p_{h}\right) \in \mathbf{P}(m)$. It is easy to check that

$$
\# P_{\mathbf{p}}\left(\mathbb{F}_{q}\right)=g_{p_{1}} \cdot g_{p_{2}} \cdots g_{p_{h}} \cdot q^{\theta(\mathbf{p})}
$$

where $\theta(\mathbf{p})=m^{2}-\sum_{i=1}^{h} \sum_{j=i}^{h} p_{i} p_{j}$. Plugging in the expression (3.3) and simplifying, we get

$$
\# P_{\mathbf{p}}\left(\mathbb{F}_{q}\right)=(-1)^{m} q^{\frac{m(m-1)}{2}} \prod_{i=1}^{h}(q)_{p_{i}}
$$

Hence

$$
-\phi(m)(q)_{m}=(-1)^{m} q^{-\frac{m(m-1)}{2}} \sum_{\mathbf{p} \in \mathbf{P}(m)}(-1)^{\ell(\mathbf{p})}\left[\begin{array}{c}
m \\
\mathbf{p}
\end{array}\right]=1,
$$

where the last equality is due to Lemma 3.2 .

3.2. Definition of the building and its basic properties. Let $\mathcal{V}$ be an $n$ dimensional vector space over $F_{\infty}$. By a basis of $\mathcal{V}$ we always mean an ordered basis. Let $C=\left(b_{1}, b_{2}, \ldots, b_{n}\right)$ be a basis of $\mathcal{V}$. The $\mathcal{O}_{\infty}$-module

$$
C_{\mathcal{O}_{\infty}}:=b_{1} \mathcal{O}_{\infty} \oplus b_{2} \mathcal{O}_{\infty} \oplus \cdots \oplus b_{n} \mathcal{O}_{\infty}
$$

is a lattice in $\mathcal{V}$. Given a lattice $L$, the group $F_{\infty}^{\times}$acts by scalar multiplications, and $x L$ is also a lattice for any $x \in F_{\infty}^{\times}$. This defines an equivalence relation on the set of lattices in $\mathcal{V}$. We denote the equivalence class of $L$ by $[L]:=\left\{x L \mid x \in F_{\infty}^{\times}\right\}$. Since $F_{\infty}$ is a local field, $[L]$ can be identified with $\left\{\pi_{\infty}^{i} L \mid i \in \mathbb{Z}\right\}$. 
Definition 3.6. The Bruhat-Tits building of $\mathrm{PGL}_{n}\left(F_{\infty}\right)$ is the simplicial complex $\mathcal{B}$ with the set of vertices $\{[L] \mid L$ is a lattice in $\mathcal{V}\}$ and the set of $i$-simplices consisting of $\left\{\left[L_{0}\right], \ldots,\left[L_{i}\right]\right\}$, such that there is $L_{j}^{\prime} \in\left[L_{j}\right]$ for each $j$ with

$$
L_{0}^{\prime} \supsetneqq L_{1}^{\prime} \supsetneqq \cdots \supsetneqq L_{i}^{\prime} \supsetneqq \pi_{\infty} L_{0}^{\prime} \text {. }
$$

$\mathcal{B}$ is $(n-1)$-dimensional. Indeed, any $i$-simplex as above produces the flag

$$
L_{0}^{\prime} / \pi_{\infty} L_{0}^{\prime} \supset L_{1}^{\prime} / \pi_{\infty} L_{0}^{\prime} \supset \cdots \supset L_{i}^{\prime} / \pi_{\infty} L_{0}^{\prime} \supset 0
$$

in the $n$-dimensional $\mathbb{F}_{q}$-vector space $L_{0}^{\prime} / \pi_{\infty} L_{0}^{\prime}$.

Fix a basis $E=\left\{e_{1}, \ldots, e_{n}\right\}$ of $\mathcal{V}$. For any $n$-tuple $i_{1}, \ldots, i_{n} \in \mathbb{Z}$ denote by $\left[i_{1}, i_{2} \ldots, i_{n}\right]$ the equivalence class of the lattice $\pi_{\infty}^{i_{1}} e_{1} \mathcal{O}_{\infty} \oplus \cdots \oplus \pi_{\infty}^{i_{n}} e_{n} \mathcal{O}_{\infty}$. The maximal subcomplex $\mathcal{A}$ of $\mathcal{B}$ having set of vertices

$$
\operatorname{Ver}(\mathcal{A})=\left\{\left[i_{1}, i_{2} \ldots, i_{n}\right] \mid i_{1}, \ldots, i_{n} \in \mathbb{Z}\right\}
$$

is called the standard apartment of $\mathcal{B}$. The maximal subcomplex $\mathcal{W}$ of $\mathcal{B}$ having set of vertices

$$
\operatorname{Ver}(\mathcal{W})=\left\{\left[i_{1}, i_{2} \ldots, i_{n}\right] \mid i_{1} \leq i_{2} \leq \cdots \leq i_{n}\right\}
$$

is called the standard Weyl chamber of $\mathcal{B}$. Note that every vertex of $\mathcal{A}$ has a unique representative of the form $\left[0, i_{2} \ldots, i_{n}\right]$.

The group $G\left(F_{\infty}\right)$ operates on the vertices of $\mathcal{B}$ by $g[L]:=[g L]$. Since $G\left(F_{\infty}\right)$ preserves the inclusions of lattices, it acts on $\mathcal{B}$.

Definition 3.7. Let $C=\left\{b_{1}, \ldots, b_{n}\right\}$ be a basis and let $[L]=\left[C_{\mathcal{O}_{\infty}}\right]$. Let $\operatorname{det}(C)$ be the determinant of the matrix having as its columns the basis elements $b_{i}$. The type of $[L]$ is an element of $\mathbb{Z} / n \mathbb{Z}$ defined by

$$
\text { Type }([L]):=\operatorname{ord}_{\infty}(\operatorname{det}(C)) \bmod n .
$$

The following lemma is well-known and is easy to prove.

Lemma 3.8. Type $([L])$ is well-defined and is invariant under the action of $\Gamma$. Each $(n-1)$-simplex of $\mathcal{B}$ has a vertex of each type. Two vertices having the same type are not adjacent in $\mathcal{B}$.

Lemma 3.9. Let $\Gamma^{\prime}$ be a subgroup of $\Gamma$, and let $s=\left\{v_{0}, \cdots, v_{m}\right\}$ be a m-simplex of $\mathcal{B}$. Then

$$
\operatorname{Stab}_{\Gamma^{\prime}}(s)=\bigcap_{i=0}^{m} \operatorname{Stab}_{\Gamma^{\prime}}\left(v_{i}\right) .
$$

Proof. Suppose $g \in \Gamma^{\prime}$. According to Lemma 3.8, the type of each $v_{i}$ differs from the type of other vertices in $s$. Since by the same lemma $g$ preserves the type of each vertex, $g\left\{v_{0}, \ldots, v_{m}\right\}=\left\{v_{0}, \ldots, v_{m}\right\}$ if and only if $g v_{i}=v_{i}$ for $0 \leq i \leq m$.

Locally, $\mathcal{B}$ describes the incidences of $\mathbb{F}_{q}$-rational linear subvarieties of $\mathbb{P}_{\mathbb{F}_{q}}^{n-1}$. More precisely, let us fix $v \in \operatorname{Ver}(\mathcal{B})$. Let $\operatorname{Star}(v)$ be the maximal simplicial subcomplex of $\mathcal{B}$ all of whose vertices are adjacent to $v$. Then the vertices of $\operatorname{Star}(v)$ are in one-to-one correspondence with the positive dimensional linear subspaces of $\mathbb{F}_{q}^{n}$. The vertices $\left\{v_{0}, \ldots, v_{i}\right\}$ form an $i$-simplex in $\operatorname{Star}(v)$ if the linear subspaces corresponding to these vertices fit into a flag. 
Example 3.10. Assume $n=3$. Then any vertex $v$ of $\mathcal{B}$ is adjacent to $2\left(q^{2}+q+1\right)$ other vertices, $v$ is a vertex of exactly $\left(q^{2}+q+1\right)(q+1) 2$-simplices in $\mathcal{B}$, any edge belongs to $(q+1) 2$-simplices. All these claims follow from the previous remark coupled with the following easy facts: $\mathbb{F}_{q}^{3}$ has $\left(q^{2}+q+1\right)$ lines and $\left(q^{2}+q+1\right)$ planes passing through the origin, any such line lies on $(q+1)$ planes and every such plane contains $(q+1)$ lines through the origin.

\subsection{The action of $\Gamma$.}

Notation 3.11. Let $0:=[0, \ldots, 0] \in \mathcal{A}$.

Lemma 3.12. Let $v=\left[i_{1}, \ldots, i_{n}\right] \in \mathcal{A}$. Then $\operatorname{Stab}_{\Gamma}(v)$ is the group of all matrices $\left(a_{j k}\right) \in \Gamma$, with $\operatorname{deg}\left(a_{j k}\right) \leq i_{k}-i_{j}$.

Proof. First, consider the stabilizer of $v$ in $G\left(F_{\infty}\right)$. Let $D=\operatorname{diag}\left(\pi_{\infty}^{i_{1}}, \ldots, \pi_{\infty}^{i_{n}}\right)$, so that $D \cdot \mathbf{0}=v$. If $g v=v$ then

$$
D^{-1} g D \in \operatorname{Stab}_{G\left(F_{\infty}\right)}(\mathbf{0})=G\left(\mathcal{O}_{\infty}\right) \cdot Z\left(F_{\infty}\right) .
$$

Hence, $\operatorname{Stab}_{G\left(F_{\infty}\right)}(v)$ is the group $\left\{\left(\pi_{\infty}^{i_{j}-i_{k}} \alpha_{j k}\right) \mid\left(\alpha_{j k}\right) \in G\left(\mathcal{O}_{\infty}\right)\right\} \cdot Z\left(F_{\infty}\right)$. Now note that $\operatorname{Stab}_{\Gamma}(v)=\operatorname{Stab}_{G\left(F_{\infty}\right)}(v) \cap \Gamma$. As $A \cap \pi_{\infty}^{m} \mathcal{O}_{\infty}$ is the set of polynomials of degree $\leq-m$, the claim follows.

Theorem 3.13. $\mathcal{W}$ is a fundamental domain for the action of $\Gamma$ on $\mathcal{B}$.

Proof. See [23].

Remark 3.14. The isomorphism $\mathcal{B} / \Gamma \cong \mathcal{W}$ can be proven using some algebraic geometry (the proof in [23] is different). The idea is the following: Let $I_{\infty} \cong$ $\mathcal{O}_{\mathbb{P}_{\mathbb{F}_{q}}}(-1)$ be the sheaf of ideals of the point $\infty=1 / T$ on $\mathbb{P}_{\mathbb{F}_{q}}^{1}$. Two vector bundles $V$ and $V^{\prime}$ on $\mathbb{P}_{\mathbb{F}_{q}}^{1}$ are said to be $I_{\infty}$-equivalent if there is $m \in \mathbb{Z}$ such that $V^{\prime} \cong I_{\infty}^{\otimes m} \otimes V$. As in [22, §II.2.1], one shows that there is a bijection between $\operatorname{Ver}(\mathcal{B} / \Gamma)$ and the set of $I_{\infty}$-equivalence classes of rank- $n$ vector bundles on $\mathbb{P}_{\mathbb{F}_{q}}$. On the other hand, by a theorem of Grothendieck every vector bundle $V$ over the projective line is a direct sum of line bundles, so can be written as

$$
I_{\infty}^{\otimes i_{1}} \oplus I_{\infty}^{\otimes i_{2}} \oplus \cdots \oplus I_{\infty}^{\otimes i_{n}}
$$

where $i_{1} \leq i_{2} \leq i_{3} \leq \cdots \leq i_{n}$, cf. [15, Cor. V.2.14]. The map

$$
I_{\infty}^{\otimes i_{1}} \oplus I_{\infty}^{\otimes i_{2}} \oplus \cdots \oplus I_{\infty}^{\otimes i_{n}} \mapsto\left[i_{1}, i_{2}, \ldots, i_{n}\right]
$$

establishes a bijection between the $I_{\infty}$-equivalence classes of rank- $n$ vector bundles and the vertices of $\mathcal{W}$.

Notation 3.15. Denote by $\mathcal{B}(\mathfrak{n})$ the $\Delta$-complex $\mathcal{B} / \Gamma(\mathfrak{n})$.

Clearly $\mathcal{B}(\mathfrak{n})$ is connected since a path between two vertices in $\mathcal{B}$ descends to a path between the images of these vertices in $\mathcal{B}(\mathfrak{n})$.

Remark 3.16. One can show that $\mathcal{B}(\mathfrak{n})$ is in fact a simplicial complex; see [7, Thm. 4.13]. (Although the running hypothesis in loc. cit. is $n=3$, the proof readily generalizes to an arbitrary $n \geq 2$.) A key intermediate fact which goes into the proof is the following:

Let $\left\{v_{0}, \ldots, v_{i}\right\}$ and $\left\{u_{0}, \ldots, u_{i}\right\}$ be $i$-simplices of $\mathcal{B}$. Suppose there are $\gamma_{0}, \ldots, \gamma_{i} \in$ $\Gamma(\mathfrak{n})$ with $\gamma_{0} v_{0}=u_{0}, \ldots, \gamma_{i} v_{i}=u_{i}$. Then there is some $\gamma \in \Gamma(\mathfrak{n})$ with $\gamma v_{0}=$ $u_{0}, \ldots, \gamma v_{i}=u_{i}$. 
This property is very specific to $\Gamma(\mathfrak{n})$ and is false for general congruence subgroups. For example, take $n=2$ and consider the quotient $\mathcal{B}^{\prime}(\mathfrak{n})$ of $\mathcal{B}$ (a tree in this case) by the Hecke congruence subgroup $\Gamma_{0}(\mathfrak{n})$. Then $\mathcal{B}^{\prime}(\mathfrak{n})$ quite often has two distinct edges joining the same two vertices, i.e., $\mathcal{B}^{\prime}(\mathfrak{n})$ is not a simplicial complex.

Nevertheless, treating $\mathcal{B}(\mathfrak{n})$ as a $\Delta$-complex will be sufficient for our purposes.

3.4. Euler-Poincaré characteristic of $\mathcal{B}(\mathfrak{n})$. Define the operators $d_{2}, d_{3}, \ldots, d_{n}$ on $\operatorname{Ver}(\mathcal{A})$ by

$$
d_{j}\left(\left[i_{1}, \ldots, i_{n}\right]\right)=\left[i_{1}, i_{2}, \ldots, i_{j-1}, i_{j}+1, i_{j+1}+1, \ldots, i_{n}+1\right] .
$$

Note that we could have defined $d_{1}$ by the same formula, but then $d_{1}$ is simply the identity map since $\left[i_{1}+m, \ldots, i_{n}+m\right]=\left[i_{1}, \ldots, i_{n}\right]$ for any $m \in \mathbb{Z}$. It is clear that $d_{j}$ 's commute with each other and any vertex of $\mathcal{W}$ can be obtained from $\mathbf{0}$ by a unique (up to permutations) sequence of $d_{j}$ 's. Let $v \in \operatorname{Ver}(\mathcal{W})$, and $v=d_{2}^{s_{2}} \ldots d_{n}^{s_{n}}(\mathbf{0})$, where $s_{j} \geq 0$ and if $s_{j}=0$ then $d_{j}^{0}$ means the identity map. Define $\operatorname{deg}_{j}(v)=s_{j}$. The map $v \mapsto d_{2}^{s_{2}} \ldots d_{n}^{s_{n}}$ gives a one-to-one correspondence between the vertices of $\mathcal{W}$ and the monomials in $d_{j}$ 's.

There is a partial ordering on the vertices of $\mathcal{W}$. If $v=\left[0, a_{2}, a_{3}, \ldots, a_{n}\right]$ and $v^{\prime}=\left[0, b_{2}, b_{3}, \ldots, b_{n}\right]$ are in $\mathcal{W}$, then we put $v \preceq v^{\prime}$ if $a_{j} \leq b_{j}$ for all $2 \leq j \leq n$, and $v \prec v^{\prime}$ if at least one of the inequalities is strict. From the definitions, it is easy to see that the vertices $\left\{v_{0}, \ldots, v_{i}\right\}$ of $\mathcal{W}$ form an $i$-simplex if and only if, up to reindexing, $v_{0} \prec v_{1} \prec \cdots \prec v_{i} \preceq d_{2}\left(v_{0}\right)$. We call $v_{0}$ the smallest vertex of $\sigma$.

For any $k \geq 0$, let $\mathcal{W}_{k}$ be the the maximal subcomplex of $\mathcal{W}$ having set of vertices

$$
\operatorname{Ver}\left(\mathcal{W}_{k}\right)=\left\{v \mid \operatorname{deg}_{2}(v) \leq k, \ldots, \operatorname{deg}_{n}(v) \leq k\right\} .
$$

Let $v$ be a vertex of $\mathcal{W}$. Denote by $K(v)$ the maximal subcomplex of $\mathcal{W}$ having set of vertices

$$
\operatorname{Ver}(K(v))=\left\{d_{2}^{s_{2}} \ldots d_{n}^{s_{n}}(v) \mid 0 \leq s_{j} \leq 1 \text { for } 2 \leq j \leq n\right\} .
$$
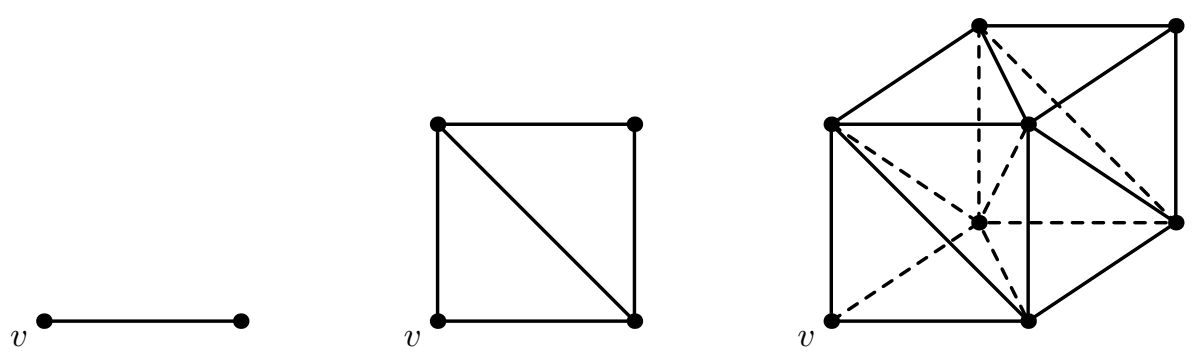

FiguRE $1 . K(v)$ for $n=2,3,4$.

For a fixed $2 \leq m \leq n$, define $K_{m}^{x}(v)$, for $x=0$ or 1 , to be the subcomplex with

$$
\operatorname{Ver}\left(K_{m}^{x}(v)\right)=\left\{d_{2}^{s_{2}} \ldots d_{n}^{s_{n}}(v) \mid 0 \leq s_{j} \leq 1 \text { for } 2 \leq j \leq n \text {, and } s_{m}=x\right\} .
$$

Let $K^{0}(v)=K(v)-\bigcup_{m=2}^{n} K_{m}^{1}(v)$ be the the set of simplices in $K(v)$ which do not completely lie in one of the $K_{m}^{1}(v)$ 's. Note that $K^{0}(v)$ is not a simplicial complex since not every face of a simplex in $K^{0}(v)$ lies in $K^{0}(v)$.

Since $\Gamma(\mathfrak{n})$ is a normal subgroup of $\Gamma, \Gamma(\mathfrak{n}) \backslash \Gamma$ is a group, which we denote $\Upsilon(\mathfrak{n})$. It is well-known that $\Upsilon(\mathfrak{n}) \cong \mathbb{F}_{q}^{\times} \ltimes \operatorname{SL}_{n}(A / \mathfrak{n})$. For a simplex $w \in \mathcal{W}$, denote the image of $\Gamma_{w}=\operatorname{Stab}_{\Gamma}(w)$ in $\Upsilon(\mathfrak{n})$ by $\overline{\Gamma_{w}}$. 


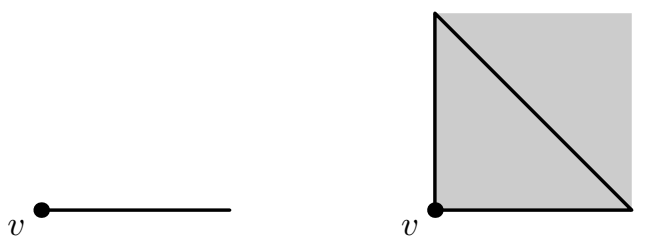

Figure 2. $K^{0}(v)$ for $n=2,3$.

Let $\mathcal{S}$ be a set of simplices in $\mathcal{W}$ (for example, a simplicial subcomplex). Define

$$
\tilde{\chi}(\mathcal{S})=\sum_{w \in \mathcal{S}}(-1)^{\operatorname{dim}(w)}\left(\# \overline{\Gamma_{w}}\right)^{-1},
$$

where the sum is over all non-oriented simplices in $\mathcal{S}$.

\section{Proposition 3.17.}

$$
\tilde{\chi}\left(K^{0}(v)\right)= \begin{cases}\phi(n), & \text { if } v=\mathbf{0} \\ 0, & \text { otherwise. }\end{cases}
$$

Proof. First we prove the claim assuming $n=2$, as this case is somewhat degenerate. If $n=2$ then $\mathcal{W}$ is the infinite half-line:

$$
[0,0]-[0,1]-[0,2]-\cdots-[0, m]-\cdots
$$

Lemma 3.12 implies that $\operatorname{Stab}_{\Gamma}(\mathbf{0})=\mathrm{GL}_{2}\left(\mathbb{F}_{q}\right)$, and $\operatorname{Stab}_{\Gamma}([0, m])$ is the group of the upper-triangular matrices $\left(\begin{array}{ll}a & b \\ 0 & d\end{array}\right)$, with $a, d \in \mathbb{F}_{q}^{\times}$and $b \in A, \operatorname{deg}(b) \leq m$. Now $K^{0}([0, m])$ consists of one 0 -dimensional simplex, namely $[0, m]$, and one 1 dimensional simplex, namely the edge joining $[0, m]$ to $[0, m+1]$. The stabilizer of this latter edge is $\operatorname{Stab}_{\Gamma}([0, m]) \cap \operatorname{Stab}_{\Gamma}([0, m+1])$, which is equal to $\operatorname{Stab}_{\Gamma}([0, m])$ when $m>0$, and is the Borel subgroup of upper-triangular matrices in $\mathrm{GL}_{2}\left(\mathbb{F}_{q}\right)$ when $m=0$. From this the claim of the proposition easily follows.

Now assume $n \geq 3$. Let $K^{00}(v)$ be the subset of $K^{0}(v)$ consisting of simplices not containing $v$. First, we show that $\tilde{\chi}\left(K^{00}(v)\right)=0$ for any $v$. In fact, we will prove a stronger statement: The set $K^{00}(v)$ can be divided into pairs of simplices $(s, \sigma)$ such that $s$ is a face of $\sigma$, the smallest vertices of $s$ and $\sigma$ are the same, $\operatorname{dim}(\sigma)=\operatorname{dim}(s)+1, \Gamma_{s}=\Gamma_{\sigma}$, and each simplex of $K^{00}(v)$ appears exactly once in some pair. (This clearly implies $\tilde{\chi}\left(K^{00}(v)\right)=0$ as the summands corresponding to $s$ and $\sigma$ cancel each other.) We proceed by induction on $n$.

When $n=3, K^{00}(v)$ consists of the 1-simplex $s=\left\{d_{2}(v), d_{3}(v)\right\}$, and the 2simplex $\sigma=\left\{d_{2}(v), d_{3}(v), d_{2} d_{3}(v)\right\}$. The smallest vertex of both $\sigma$ and $s$ is $d_{3}(v)$. Using Lemma 3.9 and Lemma 3.12, one easily checks that $\Gamma_{s}=\Gamma_{\sigma}$.

Assume we have proven the claim for $n-1$. Let $v^{\prime} \in \operatorname{Ver}(K(v))$ be $v^{\prime}=$ $d_{2}^{s_{2}} \ldots d_{n}^{s_{n}}(v)$. Define $\pi_{2}\left(v^{\prime}\right):=d_{3}^{s_{3}} \cdots d_{n}^{s_{n}}(v)$. This gives a map

$$
\pi_{2}: \operatorname{Ver}(K(v)) \rightarrow \operatorname{Ver}\left(K_{2}^{0}(v)\right)
$$

We claim that $\pi_{2}$ is a simplicial map from $K(v)$ onto $K_{2}^{0}(v)$, i.e., if $\sigma=\left\{v_{0}, \ldots, v_{i}\right\}$ is an $i$-simplex in $K(v)$ then the vertices (modulo repetitions) $\left\{\pi_{2}\left(v_{0}\right), \ldots, \pi_{2}\left(v_{i}\right)\right\}$ form a simplex in $K_{2}^{0}(v)$. Since $\sigma$ is a simplex, we can assume $v_{0} \prec v_{1} \prec \cdots \prec$ $v_{i} \preceq d_{2}\left(v_{0}\right)$. We need to show that the vertices in $\pi_{2}(\sigma)$ can be arranged to satisfy similar inequalities. We can assume there is $v_{j}$ with $\operatorname{deg}_{2}\left(v_{j}\right)=1$; otherwise $\pi_{2}$ is 
the identity on $\sigma$ and the claim is trivial. Let $j$ be the smallest index for which $\operatorname{deg}_{2}\left(v_{j}\right)=1$. Then $\operatorname{deg}_{2}\left(v_{k}\right)=1$ for any $k \geq j$. If $j=0$ then clearly

$$
\pi_{2}\left(v_{0}\right) \prec \pi_{2}\left(v_{1}\right) \cdots \prec \pi_{2}\left(v_{i}\right) \preceq d_{2} \pi_{2}\left(v_{0}\right) .
$$

Now assume $j>0$. We claim that

$\pi_{2}\left(v_{j}\right) \prec \pi_{2}\left(v_{j+1}\right) \prec \cdots \prec \pi_{2}\left(v_{i}\right) \preceq \pi_{2}\left(v_{0}\right) \prec \pi_{2}\left(v_{1}\right) \cdots \prec \pi_{2}\left(v_{j-1}\right) \preceq d_{2} \pi_{2}\left(v_{j}\right)=v_{j}$.

Since $\pi_{2}\left(v_{k}\right)=v_{k}$ for $k \leq j-1$, all the inequalities are obvious except possibly for $\pi_{2}\left(v_{i}\right) \preceq \pi_{2}\left(v_{0}\right)=v_{0}$, which is true since $v_{i} \preceq d_{2}\left(v_{0}\right)$. (Note that $\pi_{3}, \pi_{4}$, etc. defined similarly to $\pi_{2}$ are not necessarily simplicial maps. Take for example, $n=4$ and consider the edge $\{[0,0,1,1],[0,1,1,2]\}$ in $K(\mathbf{0})$. Then $\pi_{3}([0,0,1,1])=\mathbf{0}$, $\pi_{3}([0,1,1,2])=[0,1,1,2]$ which are not adjacent. $)$

Suppose $\sigma$ is an $i$-simplex in $K(v)$. By the previous paragraph $\pi_{2}(\sigma)$ is a simplex. Moreover, since the kernel of $\pi_{2}$ extended to the ambient $\mathbb{R}$-vector space containing $K(v)$ is 1-dimensional, $\pi_{2}(\sigma)$ is either $i$ or $(i-1)$-dimensional. If $\operatorname{dim}\left(\pi_{2}(\sigma)\right)=i-1$, then $\sigma$ has an $(i-1)$-dimensional face $s$ such that $\sigma=\left\{s, d_{2}\left(v^{\prime}\right)\right\}$ for some $v^{\prime} \in$ $\operatorname{Ver}(s)$. It is easy to check that this face can be uniquely characterized as follows: If $\sigma=\left\{v_{0}, \ldots, v_{i}\right\}$ with $v_{0} \prec \cdots \prec v_{i}$, then $s=\left\{v_{0}, \ldots, v_{i-1}\right\}$ and $v_{i}=d_{2}\left(v_{0}\right)$. We call $s$ the $d_{2}$-bottom of $\sigma$. On the other hand, if $s$ is an $i$-simplex in $K^{0}(v)$ and $\operatorname{dim}\left(\pi_{2}(s)\right)=i$ then $s$ is the $d_{2}$-bottom of a unique $\sigma$ in $K^{0}(v)$. Indeed, let $s=\left\{v_{0}, \ldots, v_{i}\right\}$ with $v_{0} \prec \cdots \prec v_{i}$. The vertex $v_{0}$ is not in $K_{2}^{1}(v)$ as otherwise $s \in K_{2}^{1}(v)$, which contradicts the assumption $s \in K^{0}(v)$. Hence $\pi_{2}\left(v_{0}\right)=v_{0}$. Now the assumption that $\operatorname{dim}\left(\pi_{2}(s)\right)=i$ implies $v_{i} \supsetneqq d_{2}\left(v_{0}\right)$. The set of vertices $\left\{v_{0}, \ldots, v_{i}, d_{2}\left(v_{0}\right)\right\}$ form an $(i+1)$-simplex $\sigma$, with $d_{2}$-bottom $s$. The previous arguments also show that $\sigma$ is the only $(i+1)$-simplex having $s$ as its $d_{2}$-bottom.

Let $(s, \sigma)$ be a pair of simplices in $K(v)$ such that $s$ is the $d_{2}$-bottom of $\sigma$. As is easy to see, if one of these simplices lies in $K^{0}(v)$ then so does the other one. Combining this with the previous paragraph, we conclude that all simplices in $K^{0}(v)$ can be divided into disjoint pairs $(s, \sigma), s$ is the $d_{2}$-bottom of $\sigma$.

Let $(s, \sigma)$ be as above. Assume either $s$ does not lie in $K_{2}^{0}(v)$, or $\operatorname{deg}_{2}(v) \geq 1$. We claim that under one of these assumptions $\Gamma_{s}=\Gamma_{\sigma}$. Let $s=\left\{v_{0}, \ldots, v_{i}\right\}$ with $v_{0} \prec \cdots \prec v_{i}$. Then $\sigma=\left\{v_{0}, \ldots, v_{i}, d_{2}\left(v_{0}\right)\right\}$. Using Lemma 3.9, we can assume $i=1$, and need to show

$$
\operatorname{Stab}_{\Gamma}\left(v_{0}\right) \cap \operatorname{Stab}_{\Gamma}\left(v_{1}\right) \subset \operatorname{Stab}_{\Gamma}\left(d_{2}\left(v_{0}\right)\right) .
$$

Since $\operatorname{deg}_{2}\left(v_{1}\right) \geq 1$, this follows from Lemma 3.12 .

Now let $\operatorname{deg}_{2}(v)=0$, and $s \in K_{2}^{0}(v)$. In this case we don't necessarily have $\Gamma_{s}=\Gamma_{\sigma}$. We will pair all simplices of $K^{00}(v)$ lying or having a codimension one face in $K_{2}^{0}(v)$ in a different way. Write $v=\left[0,0, i_{3}, \ldots, i_{n}\right]$. Let $v^{\prime}=\left[0, i_{3}, \ldots, i_{n}\right]$. We have a canonical isomorphism of simplicial complexes $K_{2}^{0}(v) \cong K\left(v^{\prime}\right)$, where the second complex is in the building of $\mathrm{PGL}_{n-1}\left(F_{\infty}\right)$, which preserves the partial ordering $\prec$ on the vertices. Denote $\Gamma^{\prime}:=\mathrm{GL}_{n-1}(A)$. By induction hypothesis, all simplices in $K^{00}\left(v^{\prime}\right)$ can be divided into disjoint pairs $\left(s^{\prime}, \sigma^{\prime}\right)$ where $s^{\prime}$ is a face of $\sigma^{\prime}$ of codimension one, $s^{\prime}$ contains the smallest vertex of $\sigma^{\prime}$, and $\Gamma_{s^{\prime}}^{\prime}=\Gamma_{\sigma^{\prime}}^{\prime}$. It is easy to check that if we consider $s^{\prime}, \sigma^{\prime}$ as simplices of $K^{00}(v)$, we still have $\Gamma_{s^{\prime}}=\Gamma_{\sigma^{\prime}}$. Let $\sigma^{\prime}=\left\{v_{0}, \ldots, v_{i}\right\}, v_{0} \prec \cdots \prec v_{i}$. The set of vertices $\sigma^{\prime \prime}=\left\{v_{0}, \ldots, v_{i}, d_{2}\left(v_{0}\right)\right\}$ forms an $(i+1)$-simplex in $K^{00}(v)$. Consider its codimension one face $s^{\prime \prime}=\left\{s^{\prime}, d_{2}\left(v_{0}\right)\right\}$. If $\Gamma_{s^{\prime}}=\Gamma_{\sigma^{\prime}}$ then by Lemma 3.9 we also have $\Gamma_{s^{\prime \prime}}=\Gamma_{\sigma^{\prime \prime}}$. By the induction hypothesis, $v_{0}$ is a vertex of $s^{\prime}$, so neither $\sigma^{\prime \prime}$ nor $s^{\prime \prime}$ is the $d_{2}$-bottom of another simplex. The 
pair $\left(s^{\prime \prime}, \sigma^{\prime \prime}\right)$ is uniquely characterized by $\left(s^{\prime}, \sigma^{\prime}\right)$. Using the induction hypothesis again, we conclude that any simplex whose $d_{2}$-bottom lies in $K_{2}^{0}(v)$ occurs in some unique pair $\left(s^{\prime \prime}, \sigma^{\prime \prime}\right)$. Since the union of the simplices in all pairs $\left(s^{\prime}, \sigma^{\prime}\right),\left(s^{\prime \prime}, \sigma^{\prime \prime}\right)$ is equal to the set of simplices of $K^{00}(v)$ lying or having a codimension one face in $K_{2}^{0}(v)$, this finishes the induction step.

Next, let $K^{01}(v)$ be the subset of $K^{0}(v)$ consisting of simplices containing $v$. Since $K^{0}(v)$ is the disjoint union of $K^{00}(v)$ and $K^{01}(v)$,

$$
\widetilde{\chi}\left(K^{0}(v)\right)=\widetilde{\chi}\left(K^{00}(v)\right)+\widetilde{\chi}\left(K^{01}(v)\right)=\tilde{\chi}\left(K^{01}(v)\right) .
$$

Denote $v_{2}:=d_{2}(v), v_{3}:=d_{3}(v), \ldots, v_{n}:=d_{n}(v)$. First, assume $v \neq \mathbf{0}$. Then $v=$ $\left[0,0, \ldots, 0, i_{h}, \ldots, i_{n}\right]$, where $i_{h} \neq 0$, for some $h \geq 2$. By Lemma 3.12, $\operatorname{Stab}_{\Gamma}(v) \subset$ $\operatorname{Stab}_{\Gamma}\left(v_{h}\right)$. Let $s \in K^{01}(v)$ be a simplex which does not have $v_{h}$ as one its vertices. Then $\left\{s, v_{h}\right\}$ is also a simplex in $K^{01}(v)$ and $\operatorname{Stab}_{\Gamma}\left(\left\{s, v_{h}\right\}\right)=\operatorname{Stab}_{\Gamma}(s)$. On the other hand, if $\sigma$ is a simplex in $K^{01}(v)$ which has $v_{h}$ as a vertex, then the unique codimension one face $s$ of $\sigma$ which does not contain $v_{h}$ is also in $K^{01}(v)$ (as $\left.v \neq v_{h}\right)$. Again we have $\operatorname{Stab}_{\Gamma}(\sigma)=\operatorname{Stab}_{\Gamma}(s)$. Summarizing, the set $K^{01}(v)$ can be divided into pairs of simplices $(\sigma, s)$ such that $s$ is a codimension one face of $\sigma, \Gamma_{s}=\Gamma_{\sigma}$ and each simplex appears exactly once in some pair. This implies $\widetilde{\chi}\left(K^{01}(v)\right)=0$.

Now let $v=\mathbf{0}$. Let $\left\{e_{1}, \ldots, e_{n}\right\}$ be our fixed basis of $\mathcal{V}$. The vertex $\mathbf{0}$ corresponds to the the lattice $L=\mathcal{O}_{\infty} e_{1} \oplus \cdots \oplus \mathcal{O}_{\infty} e_{n}$ in $\mathcal{V}$, and $v_{j}$ corresponds to the sublattice of $L$ :

$$
\mathcal{O}_{\infty} e_{1} \oplus \cdots \oplus \mathcal{O}_{\infty} e_{j-1} \oplus \pi_{\infty} \mathcal{O}_{\infty} e_{j} \oplus \cdots \oplus \pi_{\infty} \mathcal{O}_{\infty} e_{n}
$$

Hence, in the $\mathbb{F}_{q}$-vector space $V:=L / \pi_{\infty} L, v$ corresponds to $V$, and $v_{j}$ corresponds to the subspace $V_{j}$ spanned by $\left\{e_{1}, \ldots, e_{j-1}\right\}$. The stabilizer $\Gamma_{\sigma}$ of the $i$-simplex $\sigma=\left\{v, v_{j_{1}}, \ldots, v_{j_{i}}\right\}$, where $i \geq 1, v \prec v_{j_{1}} \prec \cdots \prec v_{j_{i}}$, is the stabilizer in $\operatorname{GL}_{n}\left(\mathbb{F}_{q}\right)$ of the flag $V_{j_{i}} \subset V_{j_{i-1}} \subset \cdots \subset V_{j_{1}} \subset V$ in $V$. This subgroup is $P_{\mathbf{p}}\left(\mathbb{F}_{q}\right)$, where

$$
\mathbf{p}=\left(j_{i}-1, j_{i-1}-j_{i}, j_{i-2}-j_{i-1}, \cdots, j_{1}-j_{2}, n-j_{1}+1\right) .
$$

Note that $\ell(\mathbf{p})=i+1$. The stabilizer of the 0 -simplex $\sigma=\{v\}$ is $P_{(n)}\left(\mathbb{F}_{q}\right)=$ $\mathrm{GL}_{n}\left(\mathbb{F}_{q}\right)$. Since we assume $\operatorname{deg}(\mathfrak{n}) \geq 1, \Gamma_{\sigma}=\overline{\Gamma_{\sigma}}$. We conclude

$$
\widetilde{\chi}\left(K^{01}(v)\right)=\sum_{\mathbf{p} \in \mathbf{P}(n)}(-1)^{\ell(\mathbf{p})-1}\left(\# P_{\mathbf{p}}\left(\mathbb{F}_{q}\right)\right)^{-1}=\phi(n),
$$

and this finishes the proof of the proposition.

Definition 3.18. We say that a vertex $v \in \operatorname{Ver}\left(\mathcal{W}_{k}\right)$ is a corner of $\mathcal{W}_{k}$ if $\operatorname{deg}_{j}(v)$ is equal either to 0 or $k$ for all $2 \leq j \leq n$. Clearly, $\mathcal{W}_{k}$ has $2^{n-1}$ corners.

Let $v$ be a corner of $\mathcal{W}_{k}$. Let $I_{0}(v) \subset\{2, \ldots, n\}$ be the set of indices $i$ such that $\operatorname{deg}_{i}(v)=0$, and let $I_{k}(v)$ be the complement of $I_{0}(v)$ in $\{2, \ldots, n\}$.

Let $\mathbb{K}(v)$ be the set of simplices of the form $\{v, s\}$, where $\operatorname{Ver}(s)$ is a subset (possibly empty) of the set $\left\{d_{i}(v) \mid i \in I_{0}(v)\right\}$. Note that $\mathbb{K}(\mathbf{0})$ is what we were denoting by $K^{01}(\mathbf{0})$ in the proof of Proposition 3.17, and $\mathbb{K}(v)=v$ if $v=d_{2}^{k} d_{3}^{k} \cdots d_{n}^{k}(\mathbf{0})$.

Proposition 3.19. With notation as above, we have

$$
\tilde{\chi}\left(\mathcal{W}_{k}\right)=\sum_{v} \tilde{\chi}(\mathbb{K}(v))
$$

where the sum is over all corners of $\mathcal{W}_{k}$. 
Proof. Let $v$ be a corner. Let $\mathcal{W}_{k}(v)$ be the maximal subcomplex of $\mathcal{W}_{k}$ having set of vertices

$$
\operatorname{Ver}\left(\mathcal{W}_{k}(v)\right)=\left\{v^{\prime} \mid v^{\prime} \in \operatorname{Ver}\left(\mathcal{W}_{k}\right), \operatorname{deg}_{j}\left(v^{\prime}\right)=k \text { if } j \in I_{k}(v)\right\} .
$$

In particular, $\mathcal{W}_{k}(\mathbf{0})=\mathcal{W}_{k}$. Let $\Xi(v)$ be the subset of corners of $\mathcal{W}_{k}$ contained in $\mathcal{W}_{k}(v)$; we denote the set of all corners of $\mathcal{W}_{k}$ by $\Xi$ (so $\Xi=\Xi(\mathbf{0})$ ). Denote by

$$
\mathcal{W}_{k}^{0}(v)=\mathcal{W}_{k}(v)-\bigcup_{\substack{y \in \Xi(v) \\ y \neq v}} \mathcal{W}_{k}(y),
$$

the set of simplices of $\mathcal{W}_{k}(v)$ which are not completely contained in one of $\mathcal{W}_{k}(y)$, $y \in \Xi(v), y \neq v$. Clearly $\mathcal{W}_{k}$ is the disjoint union $\coprod_{v \in \Xi} \mathcal{W}_{k}^{0}(v)$. Hence

$$
\widetilde{\chi}\left(\mathcal{W}_{k}\right)=\sum_{v \in \Xi} \widetilde{\chi}\left(\mathcal{W}_{k}^{0}(v)\right)
$$

Next, since $\mathcal{W}_{k}^{0}(\mathbf{0})$ is the disjoint union $\coprod_{v \in \mathcal{W}_{k-1}} K^{0}(v)$, Proposition 3.17 gives

$$
\widetilde{\chi}\left(\mathcal{W}_{k}^{0}(\mathbf{0})\right)=\widetilde{\chi}(\mathbb{K}(\mathbf{0})) \text {. }
$$

Note that each $\mathcal{W}_{k}^{0}(v), v \neq \mathbf{0}$, is isomorphic to $\mathcal{W}_{k}^{0}(\mathbf{0})$ in the building of $\operatorname{PGL}_{m}\left(F_{\infty}\right)$ for some $m<n$, so one can adapt the argument for $v=\mathbf{0}$ to an arbitrary corner (essentially by induction) to show that $\widetilde{\chi}\left(\mathcal{W}_{k}^{0}(v)\right)=\widetilde{\chi}(\mathbb{K}(v))$ for any $v \in \Xi$. Combined with (3.4), this proves the proposition.

For $v \in \Xi$, let $\mathbf{p}_{v}:=\mathbf{p}\left(I_{0}(v)\right) \in \mathbf{P}(n)$. This gives a one-to-one correspondence between the corners of $\mathcal{W}_{k}$ and the elements of $\mathbf{P}(n)$. For $\mathbf{p}=\left(p_{1}, \ldots, p_{h}\right) \in \mathbf{P}(n)$, let

$$
\theta(\mathbf{p})=n^{2}-\sum_{i=1}^{h} \sum_{j=i}^{h} p_{i} p_{j}=\sum_{i<j} p_{i} p_{j} \quad \text { and } \quad \Phi(\mathbf{p})=\phi\left(p_{1}\right) \cdots \phi\left(p_{h}\right) .
$$

Proposition 3.20. Let $v$ be a corner of $\mathcal{W}_{k}$. Let $\mathbf{p}_{v}$ be the corresponding partition of $n$. Let $d=\operatorname{deg}(\mathfrak{n})$. Assume $k \geq d-1$. Then

$$
\widetilde{\chi}(\mathbb{K}(v))=\Phi\left(\mathbf{p}_{v}\right) \cdot q^{-d \cdot \theta\left(\mathbf{p}_{v}\right)} .
$$

Proof. Given a partition $\mathbf{p}$, let us denote by $G_{\mathbf{p}}$ the group $M_{\mathbf{p}}\left(\mathbb{F}_{q}\right) U_{\mathbf{p}}(A / \mathfrak{n})$.

Let $\sigma$ be an $i$-simplex in $\mathbb{K}(v)$. Write $\sigma=\left\{v, d_{j_{1}}(v), \ldots, d_{j_{i}}(v)\right\}$, where $j_{1}, \ldots, j_{i} \in$ $I_{0}(v)$. Let

$$
\mathbf{p}_{\sigma}:=\mathbf{p}\left(I_{0}(v)-\left\{j_{1}, \ldots, j_{i}\right\}\right) .
$$

Using Lemma 3.12, it is easy to check that if $k \geq d-1$ then

$$
\overline{\Gamma_{\sigma}}=G_{\mathbf{p}_{v}} \cap G_{\mathbf{p}_{\sigma}} .
$$

Now the claim of the proposition follows from a simple calculation (which we omit), similar to the ones we already carried out in this section.

Theorem 3.21. Let $d=\operatorname{deg}(\mathfrak{n})$. Then

$$
\chi(\mathcal{B}(\mathfrak{n}))=[\Gamma: \Gamma(\mathfrak{n})] \sum_{\mathbf{p} \in \mathbf{P}(n)} \Phi(\mathbf{p}) \cdot q^{-d \theta(\mathbf{p})} .
$$


Proof. Denote by $\mathcal{B}_{k}(\mathfrak{n})$ the subcomplex of $\mathcal{B}(\mathfrak{n})$ which maps onto $\mathcal{W}_{k}$ under the quotient map $\mathcal{B}(\mathfrak{n}) / \Upsilon(\mathfrak{n}) \stackrel{\sim}{\longrightarrow} \mathcal{W}$. Let $w \in \mathcal{W}_{k}$ be an $i$-simplex. The number of $i$-simplices in $\mathcal{B}(\mathfrak{n})$ which map to $w$ is equal to $\left[\Upsilon(\mathfrak{n}): \overline{\Gamma_{w}}\right]$. Hence, using Euler's formula,

$$
\chi\left(\mathcal{B}_{k}(\mathfrak{n})\right)=\sum_{w \in \mathcal{W}_{k}}(-1)^{\operatorname{dim}(w)}\left[\Upsilon(\mathfrak{n}): \overline{\Gamma_{w}}\right]=[\Gamma: \Gamma(\mathfrak{n})] \cdot \widetilde{\chi}\left(\mathcal{W}_{k}\right) .
$$

From Proposition 3.19 and Proposition 3.20, we conclude that there is an equality $\chi\left(\mathcal{B}_{k}(\mathfrak{n})\right)=\chi\left(\mathcal{B}_{d-1}(\mathfrak{n})\right)$ for any $k \geq d-1$. On the other hand, since $\mathcal{B}_{k}(\mathfrak{n}) \subset$ $\mathcal{B}_{k+1}(\mathfrak{n})$ and $\bigcup_{k=1}^{\infty} \mathcal{B}_{k}(\mathfrak{n})=\mathcal{B}(\mathfrak{n})$, Harder's results in [12], [14] imply that there are isomorphism $H_{i}(\mathcal{B}(\mathfrak{n})) \cong H_{i}\left(\mathcal{B}_{k}(\mathfrak{n})\right), 0 \leq i \leq n-1$, when $k$ is large enough. Thus $\chi(\mathcal{B}(\mathfrak{n}))=\chi\left(\mathcal{B}_{d-1}(\mathfrak{n})\right)$ (see also [16, Prop. 3.33]). Now the formula of the theorem follows from Propositions 3.19 and 3.20.

Example 3.22. Let $n=2$. We compute $\chi(\mathcal{B}(\mathfrak{n}))$ by applying the formula in Theorem 3.21. The ordered partitions of 2 are $(2)$ and $(1,1)$. Now $\theta((2))=0, \theta((1,1))=1$, and by Proposition 3.5

$$
\phi(1)=\frac{1}{q-1} \quad \text { and } \quad \phi(2)=\frac{-1}{\left(q^{2}-1\right)(q-1)} .
$$

Hence

$$
\frac{\chi(\mathcal{B}(\mathfrak{n}))}{[\Gamma: \Gamma(\mathfrak{n})]}=\frac{-1}{\left(q^{2}-1\right)(q-1)}+\frac{1}{(q-1)^{2} q^{d}}=\frac{1}{(q-1)^{2}}\left(\frac{1}{q^{d}}-\frac{1}{q+1}\right) .
$$

This recovers the formula in [11, Cor. 5.8]; see also [22, Ch. II].

Example 3.23. Let $n=3$. There are four ordered partitions of 3 , namely $(3),(2,1)$, $(1,2),(1,1,1)$. We have

$$
\theta((3))=0, \theta((2,1))=\theta((1,2))=2, \theta((1,1,1))=3 .
$$

Next, $\phi(3)=\left[\left(q^{3}-1\right)\left(q^{2}-1\right)(q-1)\right]^{-1}$. Thus,

$$
\begin{gathered}
\frac{\chi(\mathcal{B}(\mathfrak{n}))}{[\Gamma: \Gamma(\mathfrak{n})]}=\phi(3)+\phi(1) \phi(2) q^{-2 d}+\phi(1)^{3} q^{-3 d} \\
=\frac{1}{(q-1)^{3}}\left(\frac{1}{\left(q^{2}+q+1\right)(q+1)}-\frac{2}{(q+1) q^{2 d}}+\frac{1}{q^{3 d}}\right) .
\end{gathered}
$$

This recovers the formula in [7, Cor. 6.11].

Remark 3.24. As we mentioned earlier, $[\Gamma: \Gamma(\mathfrak{n})]=(q-1) \# \operatorname{SL}_{n}(A / \mathfrak{n})$. Therefore, this number can be expressed in terms of $q$ and the degrees of primary components of $\mathfrak{n}$. For example, assume $\mathfrak{n}=\mathfrak{p}_{1} \mathfrak{p}_{2} \cdots \mathfrak{p}_{s}$ is square-free $\left(\mathfrak{p}_{i}\right.$ are prime). Let $d_{i}:=$ $\operatorname{deg}\left(\mathfrak{p}_{i}\right)$. Then

$$
[\Gamma: \Gamma(\mathfrak{n})]=(q-1) \frac{\prod_{i=1}^{s} \prod_{j=0}^{n-1}\left(q^{n d_{i}}-q^{j d_{i}}\right)}{\prod_{i=1}^{s}\left(q^{d_{i}}-1\right)} .
$$

\section{Corollary 3.25.}

$$
\lim _{\operatorname{deg}(\mathfrak{n}) \rightarrow \infty} \frac{\chi(\mathcal{B}(\mathfrak{n}))}{[\Gamma: \Gamma(\mathfrak{n})]}=\phi(n)
$$

Proof. This is a trivial consequence of Theorem 3.21 . 
Let $\zeta_{F}(s)=\prod_{v}\left(1-q_{v}^{-s}\right)^{-1}$ be the zeta-function of $F$. (Here the product is over all valuations of $F$.) It is well-know (and easy to show) that

$$
\zeta_{F}(s)=\frac{1}{\left(1-q^{-s}\right)\left(1-q^{1-s}\right)} .
$$

Let

$$
\zeta_{F, \infty}(s):=\prod_{v \neq \infty}\left(1-q_{v}^{-s}\right)^{-1}=\frac{1}{1-q^{1-s}}
$$

For any $m \geq 1$

$$
\prod_{i=0}^{m-1} \zeta_{F, \infty}(-i)=\frac{1}{(1-q)\left(1-q^{2}\right) \cdots\left(1-q^{m}\right)}=-\phi(m)
$$

Hence Theorem 3.21 relates the Euler-Poincaré characteristic of $\mathcal{B}(\mathfrak{n})$ (equiv. the Euler-Poincaré characteristic of $\Gamma(\mathfrak{n})$ ) to the values at negative integers of the partial zeta-function $\zeta_{F, \infty}$. This can be interpreted as a Gauss-Bonnet type formula; see [21]. The contributions of the "cusps" correspond to the contributions of the corners different from $\mathbf{0}$. Corollary 3.25 says that these contributions are minuscule when $\operatorname{deg}(\mathfrak{n})$ is large.

3.5. Harder's theorem. To state the main result of this subsection, we need to recall some notions from the theory of automorphic forms.

For a subset $I \subseteq\{2, \ldots, n\}$ let $V_{I}$ be the vector space of $\mathbb{C}$-valued locally constant functions on $G\left(F_{\infty}\right)$ which are left $P_{I}\left(F_{\infty}\right)$-invariant. Let $\left(V_{I}, \rho_{I}\right)$ be the representation $\rho_{I}: G\left(F_{\infty}\right) \rightarrow \operatorname{End}\left(V_{I}\right)$ of $G\left(F_{\infty}\right)$ induced by right translations on $P_{I}\left(F_{\infty}\right) \backslash G\left(F_{\infty}\right)$. Each $\left(V_{I}, \rho_{I}\right)$ is a sub-representation of $\left(V_{\emptyset}, \rho_{\emptyset}\right)$ since any $P_{I}\left(F_{\infty}\right)$ invariant function is automatically $B\left(F_{\infty}\right)$-invariant $\left(B=P_{\emptyset}\right)$. The special (or Steinberg) representation of $G\left(F_{\infty}\right)$ is the representation on the space $V_{\emptyset} / \sum_{I \neq \emptyset} V_{I}$. We will denote this representation by $\mathrm{Sp}$.

Let $\mathcal{K}$ be an open subgroup of $G(\mathcal{O})$. An automorphic cusp form for $\mathcal{K}$ is a $\mathbb{C}$ valued function $\varphi$ on $G(\mathbb{A})$ which is left $G(F)$-invariant, right $\mathcal{K} \cdot Z\left(F_{\infty}\right)$-invariant and which satisfies the condition:

$$
\int_{U_{I}(F) \backslash U_{I}(\mathbb{A})} \varphi(u g) d u=0
$$

for each $I$ and $g \in G(\mathbb{A})$ (here $d u$ is a normalized Haar measure on the compact group $\left.U_{I}(F) \backslash U_{I}(\mathbb{A})\right)$. Denote the $\mathbb{C}$-vector space of automorphic cusp forms for $\mathcal{K}$ by $W(\mathcal{K})$.

We say that the cusp form $\varphi$ is special at $\infty$ if the right $G\left(F_{\infty}\right)$-translates of $\varphi$ generate a $G\left(F_{\infty}\right)$-module isomorphic with a direct sum of a finite number of copies of Sp. Denote the subspace of $W(\mathcal{K})$ spanned by the cusp forms which are special at $\infty$ by $W_{\mathrm{sp}}(\mathcal{K})$.

Let $\mathcal{I}$ be the Iwahori subgroup of $G\left(F_{\infty}\right)$. By definition, $\mathcal{I}$ is the inverse image of $B\left(\mathbb{F}_{\infty}\right)$ under the reduction modulo $\mathfrak{p}_{\infty}$ homomorphism $G(\mathcal{O}) \rightarrow G\left(\mathbb{F}_{\infty}\right)$. Let $\mathfrak{n}$ be an ideal in $A$. For a place $v \neq \infty$ of $F$ let $\mathfrak{n}_{v}$ be the ideal generated by $\mathfrak{n}$ in $\mathcal{O}_{v}$ under the injection $A \rightarrow \mathcal{O}_{v}$. Let $\mathcal{K}(\mathfrak{n})_{f}=\prod_{v \neq \infty} \mathcal{K}\left(\mathfrak{n}_{v}\right)$. Note that $\mathcal{K}(\mathfrak{n})_{f}$ is the adelic version of $\Gamma(\mathfrak{n})$, and in fact, $\Gamma(\mathfrak{n})=G(F) \cap \mathcal{K}(\mathfrak{n})_{f}$. Let $\mathcal{K}(\mathfrak{n})=\mathcal{K}(\mathfrak{n})_{f} \times \mathcal{I}$. Denote $W_{\mathrm{sp}}(\mathcal{K}(\mathfrak{n}))$ simply by $W_{\mathrm{sp}}(\mathfrak{n})$. 
Theorem 3.26 (Harder). The space $W_{\mathrm{sp}}(\mathfrak{n})$ is finite dimensional, and $\operatorname{dim}_{\mathbb{C}} W_{\mathrm{sp}}(\mathfrak{n})=\left[(A / \mathfrak{n})^{\times}: \mathbb{F}_{q}^{\times}\right] \cdot \operatorname{dim}_{\mathbb{Q}} H_{n-1}(\mathcal{B}(\mathfrak{n}))$.

For all $0<i<n-1$,

$$
H_{i}(\mathcal{B}(\mathfrak{n}))=0 .
$$

We briefly indicate the ideas which go into the proof of this deep result. For the proof itself see [14] (and also [12], [13]).

First, one shows that $H^{i}(\mathcal{B}(\mathfrak{n}))$ is canonically isomorphic to $H^{i}(\Gamma(\mathfrak{n}), \mathbb{Q})$ for any $0 \leq i \leq n-1$; the argument is outlined in $[21, \S 1.6$. Rem. 1]. For a discrete cocompact subgroup $\Gamma^{\prime}$ of $G\left(F_{\infty}\right)$, Serre conjectured that $H^{i}\left(\Gamma^{\prime}, \mathbb{Q}\right)$ vanish for $0<i<n-1$. This conjecture was initially proven by Garland [6], under the assumption that $q$ is large enough, and by Casselman [2] in general. Garland's argument relates the vanishing of cohomology groups to the estimates of the eigenvalues of a certain combinatorial Laplace operator; Casselman's argument uses the theory of admissible representations of $G\left(F_{\infty}\right)$. For a non-cocompact congruence subgroup of $G\left(\mathcal{O}_{\infty}\right)$, such as $\Gamma(\mathfrak{n})$, the vanishing of the middle cohomology groups was proven by Harder, using representation-theoretic methods similar to Casselman's.

Now we discuss the first part of Theorem 3.26. Let $\mathcal{K}$ be an open subgroup of $G(\mathcal{O})$. Since $G(\mathcal{O})$ is compact, $\mathcal{K}$ has finite index in $G(\mathcal{O})$. Write $\mathcal{K}=\mathcal{K}_{f} \times \mathcal{K}_{\infty}$, where $\mathcal{K}_{f}$ is an open subgroup of $G\left(\mathcal{O}_{f}\right)$ and $\mathcal{K}_{\infty}$ is an open subgroup of $G\left(\mathcal{O}_{\infty}\right)$. As results from the strong approximation theorem for $\mathrm{SL}_{n}$, the determinant induces a bijection

$$
G(F) \backslash G\left(\mathbb{A}_{f}\right) / \mathcal{K}_{f} \stackrel{\cong}{\longrightarrow} F^{\times} \backslash \mathbb{A}_{f}^{\times} / \operatorname{det} \mathcal{K}_{f},
$$

where $G(F)$ is embedded diagonally into $G(\mathbb{A})$. It is well-known that

$$
F^{\times} \backslash \mathbb{A}_{f}^{\times} / \mathcal{O}_{f}^{\times} \cong \operatorname{Pic}(A)=1
$$

We conclude that the double coset space on the left-hand side of (3.5) is finite, as $\operatorname{det} \mathcal{K}_{f}$ has finite index in $\mathcal{O}_{f}^{\times}$. Let $S$ denote a set of representatives of this finite coset space, and for $x \in S$ let $\Gamma_{x}:=G(F) \cap x \mathcal{K}_{f} x^{-1}$, where the intersection takes place in the group $G\left(\mathbb{A}_{f}\right)$. Each $\Gamma_{x}$ is an arithmetic subgroup of $G(F)$. We get the bijection

$$
G(F) \backslash G(\mathbb{A}) / \mathcal{K} Z\left(F_{\infty}\right) \stackrel{\cong}{\longrightarrow} \bigsqcup_{x \in S} \Gamma_{x} \backslash G\left(F_{\infty}\right) / \mathcal{K}_{\infty} Z\left(F_{\infty}\right)
$$

Since the stabilizer of a maximal flag in $\mathbb{F}_{\infty}^{n}$ is isomorphic to $B\left(\mathbb{F}_{\infty}\right)$, the stabilizer in $G\left(F_{\infty}\right)$ of an oriented $(n-1)$-simplex of $\mathcal{B}$ is isomorphic to $\mathcal{I}$. Therefore,

$$
\operatorname{PGL}_{n}\left(F_{\infty}\right) / \mathcal{I} \cong S_{n-1}(\mathcal{B}) \text {, }
$$

and

$$
G(F) \backslash G(\mathbb{A}) / \mathcal{K}(\mathfrak{n}) Z\left(F_{\infty}\right) \stackrel{\cong}{\longrightarrow} \bigsqcup_{x \in S} S_{n-1}\left(\mathcal{B}(\mathfrak{n})_{x}\right),
$$

where $\mathcal{B}(\mathfrak{n})_{x}$ denotes the quotient of $\mathcal{B}$ by $\Gamma(\mathfrak{n})_{x}:=G(F) \cap x \mathcal{K}(\mathfrak{n})_{f} x^{-1}$. Note that all $\mathcal{B}(\mathfrak{n})_{x}$ are isomorphic to $\mathcal{B}(\mathfrak{n})_{1}=\mathcal{B}(\mathfrak{n})$. Next, it is not hard to check that

$$
F^{\times} \backslash \mathbb{A}_{f}^{\times} / \operatorname{det} \mathcal{K}(\mathfrak{n})_{f} \cong(A / \mathfrak{n})^{\times} / \mathbb{F}_{q}^{\times},
$$

cf. $[17,(6.6)]$. Hence $\# S=\left[(A / \mathfrak{n})^{\times}: \mathbb{F}_{q}^{\times}\right]$. The upshot is that

$$
W_{\mathrm{sp}}(\mathfrak{n}) \stackrel{\cong}{\longrightarrow} \bigoplus_{x \in(A / \mathfrak{n})^{\times} / \mathbb{F}_{q}^{\times}} H_{!}^{n-1}(\mathcal{B}, \mathbb{C})^{\Gamma(\mathfrak{n})_{x}},
$$


where $H_{!}^{n-1}(\mathcal{B}, \mathbb{C})^{\Gamma(\mathfrak{n})_{x}}$ is a space of $\mathbb{C}$-valued functions on the oriented $(n-1)$ simplices of $\mathcal{B}$ which satisfy some conditions. These conditions turn out to be the following: $f$ is in $H_{!}^{n-1}(\mathcal{B}, \mathbb{C})^{\Gamma(\mathfrak{n})_{x}}$ if and only if (i) $f$ is a cochain; (ii) $f$ is harmonic, which means $f$ is in the kernel of a certain operator $\delta$ acting on the cochains, cf. [6]; (iii) $f$ is $\Gamma(\mathfrak{n})_{x}$-invariant and has finite support modulo $\Gamma(\mathfrak{n})_{x}$. The final step consists of showing

$$
H_{!}^{n-1}(\mathcal{B}, \mathbb{C})^{\Gamma(\mathfrak{n})_{x}} \cong H^{n-1}\left(\mathcal{B}(\mathfrak{n})_{x}, \mathbb{C}\right)
$$

which is a non-archimedean version of Hodge decomposition.

\section{Corollary 3.27 .}

$$
\lim _{\operatorname{deg}(\mathfrak{n}) \rightarrow \infty} \frac{\operatorname{dim}_{\mathbb{C}} W_{\mathrm{sp}}(\mathfrak{n})}{\left[(A / \mathfrak{n})^{\times}: \mathbb{F}_{q}^{\times}\right] \cdot[\Gamma: \Gamma(\mathfrak{n})]}=\frac{1}{(q-1)\left(q^{2}-1\right) \cdots\left(q^{n}-1\right)} .
$$

Proof. First of all, Theorem 3.26 implies

$$
\chi(\mathcal{B}(\mathfrak{n}))=1+(-1)^{n-1} \operatorname{dim}_{\mathbb{Q}} H_{n-1}(\mathcal{B}(\mathfrak{n})) .
$$

(Recall that $\mathcal{B}(\mathfrak{n})$ is connected, so $H_{0}(\mathcal{B}(\mathfrak{n})) \cong \mathbb{Q}$.) The rest is a trivial consequence of Corollary 3.25 and the first part of Harder's theorem.

3.6. Cusps. This subsection plays no role in what follows after it and can be skipped.

We saw that $\mathcal{B}(\mathfrak{n})$ is an infinite complex, but at the same time all the information about its homology is contained in a finite subcomplex $\mathcal{B}_{k}(\mathfrak{n})$, for $k$ large enough. Hence one would expect that the complement of such $\mathcal{B}_{k}(\mathfrak{n})$ in $\mathcal{B}(\mathfrak{n})$ has a simple simplicial structure, more or less independent of $k \gg 0$. Let us denote the minimal simplicial subcomplex of $\mathcal{B}(\mathfrak{n})$ containing $\mathcal{B}(\mathfrak{n})-\mathcal{B}_{k}(\mathfrak{n})$ by $\mathcal{B}_{k}^{c}(\mathfrak{n})$.

First, recall what happens for $n=2$, cf. [22, II.2.3]. One shows that $\mathcal{B}_{k}^{c}(\mathfrak{n})$, $k \geq \operatorname{deg}(\mathfrak{n})$, is a disjoint union of a finite number of $\mathcal{W}$ 's, i.e., is a disjoint union of a finite number of infinite half-lines. These half-lines are called cusps, and there is a formula for their number in terms of prime divisors of $\mathfrak{n}$.

Now let $n \geq 2$ be arbitrary. The previous paragraph suggests

Definition 3.28. Let $d=\operatorname{deg}(\mathfrak{n})$. A cusp of $\mathcal{B}(\mathfrak{n})$ is a connected component of $\mathcal{B}_{d}^{c}(\mathfrak{n})$.

We give one example, which shows that the simplicial structure of the cusps for $n \geq 3$ is more complicated than one would naively expect in analogy with $n=2$. A similar example for $n=3$ is discussed in [7, p. 64].

Let $\mathfrak{n}=(T)$. Let $m=\# \Upsilon(T)$, and let $g_{1}, g_{2}, \ldots, g_{m}$ be the elements of $\Upsilon(T)=$ $\mathrm{GL}_{n}\left(\mathbb{F}_{q}\right)$. Take a disjoint union of $m$ copies of $\mathcal{W}$ indexed by $g_{i}$ 's: $\mathcal{W}_{g_{1}}, \ldots, \mathcal{W}_{g_{m}}$. To obtain $\mathcal{B}(T)$ one glues $\mathcal{W}_{g_{i}}$ 's as follows. Let $v$ be a vertex of $\mathcal{W}$. We glue $\mathcal{W}_{g_{i}}$ and $\mathcal{W}_{g_{j}}$ at $v$ if and only if $g_{i}$ and $g_{j}$ have the same image in $G\left(\mathbb{F}_{q}\right) / \overline{\Gamma_{v}}$.

Let $S$ be the set of simplices in $\mathcal{W}$ having $\mathbf{0}$ as a vertex. As in the proof of Proposition 3.17 , let $v_{2}=d_{2}(\mathbf{0}), \ldots, v_{n}=d_{n}(\mathbf{0})$. Let $\sigma=\left\{\mathbf{0}, v_{j_{1}}, \ldots, v_{j_{i}}\right\}$ be an $i$-simplex in $S$. Let $\mathfrak{F}_{\sigma}$ be the subcomplex of $\mathcal{W}$ with

$$
\operatorname{Ver}\left(\mathfrak{F}_{\sigma}\right)=\left\{d_{j_{1}}^{s_{1}} \cdots d_{j_{i}}^{s_{i}}(\mathbf{0}) \mid s_{1}, \ldots, s_{i} \geq 0\right\} .
$$

Lemma 3.29. $\mathcal{W}_{g}$ and $\mathcal{W}_{h}$ in $\mathcal{B}(T)$ are glued along $\mathfrak{F}_{\sigma}$ if and only if they are glued along $\sigma$. 
Proof. First of all, as we mentioned (Remark 3.16), $\mathcal{B}(T)$ is a simplicial complex, so $\mathcal{W}_{g}$ and $\mathcal{W}_{h}$ in $\mathcal{B}(T)$ are glued along a simplex if and only if they are glued at the vertices of that simplex. Therefore, we need to show that if $\mathcal{W}_{g}$ and $\mathcal{W}_{h}$ are glued at $\mathbf{0}, v_{j_{1}}, \ldots, v_{j_{i}}$ then they are glued at any $v \in \operatorname{Ver}\left(\mathfrak{F}_{\sigma}\right)$.

As is easy to check, if $g$ and $h$ have the same image in $G\left(\mathbb{F}_{q}\right) / \overline{\Gamma_{v^{\prime}}}$ and $G\left(\mathbb{F}_{q}\right) / \overline{\Gamma_{v^{\prime \prime}}}$ then they have the same image also in $G\left(\mathbb{F}_{q}\right) /\left(\overline{\Gamma_{v^{\prime}}} \cap \overline{\Gamma_{v^{\prime \prime}}}\right)$. It is also easy to check that for any $v \neq \mathbf{0} \in \operatorname{Ver}\left(\mathfrak{F}_{\sigma}\right), \overline{\Gamma_{v}}$ contains $\overline{\Gamma_{v_{j_{1}}}} \cap \cdots \cap \overline{\Gamma_{v_{j_{i}}}}$. Combined, these two facts imply the claim.

Using the previous lemma, we conclude that in $\mathcal{B}(T)$ we have $\left[G\left(\mathbb{F}_{q}\right): B\left(\mathbb{F}_{q}\right)\right]$ distinct copies of $\mathcal{W}$. Each such $\mathcal{W}$ is glued along $\sigma=\left\{\mathbf{0}, v_{j_{1}}, \ldots, v_{j_{i}}\right\}$ to $\left[\overline{\Gamma_{\sigma}}\right.$ : $B\left(\mathbb{F}_{q}\right)$ ] other $\mathcal{W}$ 's. (Note that $\overline{\Gamma_{\sigma}}$ is a standard parabolic subgroup in $G\left(\mathbb{F}_{q}\right)$.) In particular, all $\mathcal{W}$ 's are glued at $\mathbf{0}$.

Lemma 3.30. If $n \geq 3$ then $\mathcal{B}_{k}^{c}(T)$ is connected for any $k \geq 0$.

Proof. To prove the lemma, we start by describing the structure of $\mathcal{B}(T)$ in terms of flags. Fix a basis $E=\left\{e_{1}, \ldots, e_{n}\right\}$ of $\mathcal{V}$ as in $\S 3.6$. The lattice in $\mathcal{V}$ corresponding to $\mathbf{0}$ is $E_{\mathcal{O}_{\infty}}$. Let $V=E_{\mathcal{O}_{\infty}} / \mathfrak{p}_{\infty} E_{\mathcal{O}_{\infty}} \cong \bigoplus_{i=1}^{n} \mathbb{F}_{q} e_{i}$, and let $V_{m}=\bigoplus_{i=1}^{m} \mathbb{F}_{q} e_{i}$. Let $\mathcal{F}$ be the maximal flag

$$
\mathcal{F}: 0 \subset V_{1} \subset V_{2} \subset \cdots \subset V_{n-1} \subset V_{n}=V .
$$

Modulo $\mathfrak{p}_{\infty}$, the vertex $\mathbf{0}$ corresponds to $V, v_{m}$ corresponds to $V_{m-1}, 2 \leq m \leq n$, and the flag of lattices in $\mathcal{V}$ for the $i$-simplex $\sigma=\left\{\mathbf{0}, v_{j_{1}}, \ldots, v_{j_{i}}\right\}$ maps to the flag

$$
\mathcal{F}_{\sigma}: 0 \subset V_{j_{1}-1} \subset \cdots \subset V_{j_{i}-1} \subset V .
$$

The map $\mathcal{W}_{g} \rightarrow g \mathcal{F}$ gives a one-to-one correspondence between the $\mathcal{W}$ 's in $\mathcal{B}(T)$ and the maximal flags in $V$ (note that the stabilizer of $\mathcal{F}$ in $G\left(\mathbb{F}_{q}\right)$ is exactly $B\left(\mathbb{F}_{q}\right)$ ). Under this correspondence, $\mathcal{W}_{g}$ is glued to $\mathcal{W}_{h}$ at $v_{i}$ if and only if

$$
g \mathcal{F}_{\left\{\mathbf{0}, v_{i}\right\}}=h \mathcal{F}_{\left\{\mathbf{0}, v_{i}\right\}} .
$$

Indeed, $g \mathcal{F}_{\left\{\mathbf{0}, v_{i}\right\}}=h \mathcal{F}_{\left\{\mathbf{0}, v_{i}\right\}}$ is equivalent to

$$
h^{-1} g \in \operatorname{Stab}_{G\left(\mathbb{F}_{q}\right)}\left(\mathcal{F}_{\left\{\mathbf{0}, v_{i}\right\}}\right)=\overline{\Gamma_{v_{i}}},
$$

and this is equivalent to $g$ and $h$ having the same image in $G\left(\mathbb{F}_{q}\right) / \overline{\Gamma_{v_{i}}}$. On the other hand, if $\mathcal{W}_{g}$ and $\mathcal{W}_{h}$ are glued at $v_{i}$, then by Lemma 3.29 they are glued along $\mathfrak{F}_{\left\{\mathbf{0}, v_{i}\right\}}$, which is an infinite half-line.

Now we claim that for any $\mathcal{W}^{\prime}$ and $\mathcal{W}^{\prime \prime}$ in $\mathcal{B}(T)$ there are $\mathcal{W}_{1}$ and $\mathcal{W}_{2}$ such that each $\mathcal{W}^{\prime} \cap \mathcal{W}_{1}, \mathcal{W}_{1} \cap \mathcal{W}_{2}, \mathcal{W}_{2} \cap \mathcal{W}^{\prime \prime}$ contains an infinite half-line. This implies the lemma, since $\mathcal{W}_{k}^{c}$ is connected. Let

$$
\mathcal{F}^{\prime}: 0 \subset V_{1}^{\prime} \subset \cdots \subset V_{n-1}^{\prime} \subset V \text { and } \mathcal{F}^{\prime \prime}: 0 \subset V_{1}^{\prime \prime} \subset \cdots \subset V_{n-1}^{\prime \prime} \subset V
$$

be the maximal flags corresponding to $\mathcal{W}^{\prime}$ and $\mathcal{W}^{\prime \prime}$, respectively. From what was said in the previous paragraph, if $V_{1}^{\prime}=V_{1}^{\prime \prime}$ then $\mathcal{W}^{\prime} \cap \mathcal{W}^{\prime \prime}$ already contains a halfline. Now suppose $V_{1}^{\prime} \neq V_{1}^{\prime \prime}$. Let $\mathcal{F}_{1}$ and $\mathcal{F}_{2}$ be some maximal flags which start as

$$
\mathcal{F}_{1}: 0 \subset V_{1}^{\prime} \subset V_{1}^{\prime}+V_{1}^{\prime \prime} \subset \cdots \quad \text { and } \mathcal{F}_{2}: 0 \subset V_{1}^{\prime \prime} \subset V_{1}^{\prime}+V_{1}^{\prime \prime} \subset \cdots
$$

Take $\mathcal{W}_{1}$ and $\mathcal{W}_{2}$ to be the $\mathcal{W}$ 's corresponding to $\mathcal{F}_{1}$ and $\mathcal{F}_{2}$, respectively. 
Remark 3.31. When $n=2$ the statement of the lemma is false. In fact $\mathcal{B}(T)$ consists of $(q+1)$ infinite half-lines, all joined at their origins, so $\mathcal{B}_{k}^{c}(T)$ is a disjoint union of $(q+1)$ infinite half-lines once $k \geq 1$.

We conclude that $\mathcal{B}(T)$ has one cusp which, as a simplicial complex, consists of $\left[G\left(\mathbb{F}_{q}\right): B\left(\mathbb{F}_{q}\right)\right]$ copies of $\mathcal{W}_{1}^{c}$ glued together in a rather complicated manner.

\section{DRINFEld MODUlar VARIETIES}

In this section we recall the definition of Drinfeld modules and Drinfeld modular schemes, and then compare the number of $\mathbb{F}_{q^{n}}$-rational points on Drinfeld modular varieties over $\mathbb{F}_{T} \cong \mathbb{F}_{q}$ to their $\ell$-adic Betti numbers.

4.1. Rational points. Let $S$ be a scheme over $A$. Denote by $\gamma$ the canonical ring homomorphism $\gamma: A \rightarrow H^{0}\left(S, \mathcal{O}_{S}\right)$. Fix some $n \in \mathbb{Z}_{>0}$. A pair $D=(\mathcal{G}, \varphi)$ consisting of an $\mathbb{F}_{q}$-vector space scheme $\mathcal{G}$ over $S$ and an $\mathbb{F}_{q}$-algebra homomorphism

$$
\begin{aligned}
\varphi: A & \rightarrow \operatorname{End}_{S}(\mathcal{G}), \\
a & \mapsto \varphi_{a}
\end{aligned}
$$

from $A$ into the ring of $\mathbb{F}_{q}$-linear $S$-endomorphisms of $\mathcal{G}$ is called a Drinfeld module of rank $n$ over $S$ if the following conditions are satisfied:

(1) the group scheme $\mathcal{G}$ is Zariski-locally isomorphic to the additive group scheme $\mathbb{G}_{a, S}$ over $S$;

(2) for each non-zero $a \in A, \varphi_{a}$ is finite flat of degree $|a|_{\infty}^{n}$;

(3) the induced action on the tangent space at the identity is via the structure $\operatorname{map} \gamma$.

The characteristic of $D$ is the image of $S$ in $\operatorname{Spec}(A)$ under $\gamma^{*}: S \rightarrow \operatorname{Spec}(A)$.

Example 4.1. When $S$ is a spectrum of a field $K$, the definition of a Drinfeld module over $S$ can be reformulated as follows. Let $K\{\tau\}$ be the non-commutative ring of polynomials in $\tau$ with coefficients in $K$, and the commutation rule $\tau \alpha=\alpha^{q} \tau$ for all $\alpha \in K$. Let $\gamma: A \rightarrow K$ be the structure homomorphism. A Drinfeld module $D$ over $K$ of rank $n$ is an $\mathbb{F}_{q}$-linear ring homomorphism $\varphi: A \rightarrow K\{\tau\}$, such that

$$
\varphi_{T}=\gamma(T)+\alpha_{1} \tau+\cdots+\alpha_{n} \tau^{n},
$$

where $\alpha_{1}, \ldots, \alpha_{n} \in K$ and $\alpha_{n} \neq 0$.

For $\mathfrak{n} \in A$, the finite flat group scheme $\operatorname{ker}\left(\varphi_{\mathfrak{n}}\right)$ over $S$ is called the $\mathfrak{n}$-torsion subgroup of $D$. The group $\varphi_{\mathfrak{n}}$ is an $A$-module via $\varphi$. If $\mathfrak{n}$ is disjoint from the characteristic of $D$, then $\varphi_{\mathfrak{n}}$ is locally constant with value $(A / \mathfrak{n})^{n}$ for the étale topology on $S$.

Definition 4.2. Assume $\mathfrak{n}$ is disjoint from the characteristic of $D$. A level $\mathfrak{n}$ structure on $D$ is an isomorphism of schemes of $(A / \mathfrak{n})$-modules over $S$

$$
\lambda:(A / \mathfrak{n})_{S}^{n} \longrightarrow \varphi_{\mathfrak{n}}
$$

where $(A / \mathfrak{n})_{S}^{n}$ is the constant scheme of $(A / \mathfrak{n})$-modules over $S$ with value $(A / \mathfrak{n})^{n}$.

Theorem 4.3 (Drinfeld). Let $\mathcal{M}^{n}(\mathfrak{n})$ be the functor which to each $A\left[\mathfrak{n}^{-1}\right]$-scheme $S$ associates the set of isomorphism classes $(D, \lambda)_{S}$ of Drinfeld A-modules $D$ of rank $n$ over $S$ with level $\mathfrak{n}$-structure $\lambda$. If $\mathfrak{n}$ has at least two distinct prime divisors then $\mathcal{M}^{n}(\mathfrak{n})$ is representable by a smooth affine $A\left[\mathfrak{n}^{-1}\right]$-scheme $M^{n}(\mathfrak{n})$ of relative dimension $(n-1)$. 
Proof. See $[4, \S 5]$ or $[18$, Ch. 1].

The group $G(A / \mathfrak{n})$ acts on the right of $M^{n}(\mathfrak{n})$. In terms of the moduli problem the action of $g \in G(A / \mathfrak{n})$ is given by

$$
g:(D, \lambda) \mapsto(D, \lambda \circ g) .
$$

If $\mathfrak{n}=\mathfrak{p}$ is a prime, then $\mathcal{M}^{n}(\mathfrak{p})$ has in general only a coarse moduli scheme. This coarse moduli scheme will be denoted by $M^{n}(\mathfrak{p})$. It can be obtained as follows. Let $\mathfrak{m}$ be a polynomial with at least two distinct prime divisors. The group $G(A / \mathfrak{m})$ is a normal subgroup of $G(A / \mathfrak{p m})$ (it is the kernel of the $\bmod \mathfrak{m}$ reduction map $G(A / \mathfrak{m p}) \rightarrow G(A / \mathfrak{p}))$. Hence $G(A / \mathfrak{m})$ acts on $M^{n}(\mathfrak{m} \mathfrak{p})$. Define $M^{n}(\mathfrak{p}):=M^{n}(\mathfrak{m p}) / G(A / \mathfrak{m})$; cf. [18, Lem. 1.4.2]. If $\mathfrak{p} \neq A$ then $M^{n}(\mathfrak{p})$ is smooth; see [18, Thm. 1.5.1]. On the contrary, $M^{n}(1)$ is not smooth if $n \geq 3$ (its compactification is the weigthed projective space $\left.\mathbb{P}_{A}\left(q-1, q^{2}-1, \ldots, q^{n}-1\right)\right)$.

From now on we assume that $\mathfrak{n} \neq A$ and $n \geq 2$.

Theorem 4.4. There is an $A\left[\mathfrak{n}^{-1}\right]$-morphism

$$
w_{\mathfrak{n}}: M^{n}(\mathfrak{n}) \rightarrow M^{1}(\mathfrak{n}),
$$

which is $G(A / \mathfrak{n})$-equivariant, in the sense that for $g \in G(A / \mathfrak{n})$

$$
w_{\mathfrak{n}} \circ g=\operatorname{det}(g) \circ w_{\mathfrak{n}} .
$$

Proof. See [24, Thm. 4.1] and [25]. The morphism $w_{\mathfrak{n}}$ is induced by an analogue of the Weil pairing for Drinfeld modules.

Let $F_{\mathfrak{n}}$ be the function field of $M^{1}(\mathfrak{n})$. From Class Field Theory it is known that $F_{\mathfrak{n}}$ is the maximal abelian extension of $F$ with conductor $\mathfrak{n}$ which is completely split at $\infty$, moreover $\operatorname{Gal}\left(F_{\mathfrak{n}} / F\right) \cong(A / \mathfrak{n})^{\times} / \mathbb{F}_{q}^{\times} ;$see $[4$, Thm. 1] and [17].

Corollary 4.5. The fibres of $w_{\mathfrak{n}}: M^{n}(\mathfrak{n}) \rightarrow M^{1}(\mathfrak{n})$ are smooth and geometrically irreducible.

Proof. From Theorem 4.4 and the above paragraph it is clear that $w_{\mathfrak{n}}$ is surjective, and moreover, $M^{1}(\mathfrak{n})$ has $\left[(A / \mathfrak{n})^{\times}: \mathbb{F}_{q}^{\times}\right]$geometrically connected components. The theory of rigid-analytic uniformization implies that $M^{n}(\mathfrak{n}), n \geq 2$, also has $\left[(A / \mathfrak{n})^{\times}: \mathbb{F}_{q}^{\times}\right]$geometrically irreducible components, each (as an analytic variety) isomorphic to $\Omega^{n} / \Gamma(\mathfrak{n})$; see $[4, \S 6]$. (Here $\Omega^{n}$ is Drinfeld's symmetric space.) The claim follows since $w_{\mathfrak{n}}$ commutes with base change to any $A\left[\mathfrak{n}^{-1}\right]$-field; see [24, Lem. $4.2]$.

Recall from Definition 1.1 that a prime $\mathfrak{p} \triangleleft A$ is admissible if $x \mapsto x^{n}$ is an automorphism of $\mathbb{F}_{\mathfrak{p}}^{\times} / \mathbb{F}_{q}^{\times}$.

Lemma 4.6. There are infinitely many admissible primes.

Proof. Let $d:=\operatorname{deg}(\mathfrak{p})$. We need to show that there are infinitely many $d$ such that $N_{d}:=\left(q^{d}-1\right) /(q-1)$ is coprime to $n$. Let $\ell$ be a prime divisor of $n$. If $\ell=p$ then $N_{d}$ is not divisible by $\ell$ for any $d \neq 0$. From now on assume $\ell \neq p$. If $q \equiv 1(\bmod \ell)$, then $N_{d}$ is divisible by $\ell$ if and only if $d \equiv 0(\bmod \ell)$. If $q \not \equiv 1(\bmod \ell)$, then $N_{d}$ is divisible by $\ell$ if and only if $q^{d} \equiv 1(\bmod \ell)$. This last congruence holds only if $(d, \ell-1) \neq 1$.

Let $\ell_{1}, \ldots, \ell_{s}$ be the prime divisors of $n$ which divide $(q-1)$. Let $p_{1}, \ldots, p_{r}$ be the prime divisors of $n$ which do not divide $(q-1)$ and are not not equal to $p$. 
From the previous paragraph, we conclude that any $\mathfrak{p}$ which has degree coprime to $\ell_{1} \cdots \ell_{s} \cdot\left(p_{1}-1\right) \cdots\left(p_{r}-1\right)$ is admissible.

Let $Z(A / \mathfrak{n}) \triangleleft G(A / \mathfrak{n})$ be the subgroup of scalar matrices. Assume $\mathfrak{n}$ is coprime to $T$. Let $M_{T}^{n}(\mathfrak{n}):=M^{n}(\mathfrak{n}) \otimes_{A[\mathfrak{n}-1]} \mathbb{F}_{T}$, and

$$
X_{\mathfrak{n}}:=\left(M_{T}^{n}(\mathfrak{n})\right) / Z(A / \mathfrak{n}) .
$$

Proposition 4.7. Assume $\mathfrak{p}$ is an admissible prime not equal to $T$. Then $X_{\mathfrak{p}}$ is a smooth, absolutely irreducible $(n-1)$-dimensional affine variety defined over $\mathbb{F}_{q}$, which is a form of one of the components of $M_{T}^{n}(\mathfrak{p})$.

Proof. By Corollary 4.5 the fibres of $M_{T}^{n}(\mathfrak{p}) \rightarrow M^{1}(\mathfrak{p}) \otimes \mathbb{F}_{T}$ are smooth and geometrically irreducible. Hence by Theorem 4.4 and [24, Lem. 4.2], the fibres of $X_{\mathfrak{p}} \rightarrow\left(M^{1}(\mathfrak{p}) \otimes \mathbb{F}_{T}\right) / \operatorname{det}\left(Z\left(\mathbb{F}_{\mathfrak{p}}\right)\right)$ are absolutely irreducible. On the other hand, since $\mathfrak{p}$ is an admissible prime, $\operatorname{det}\left(Z\left(\mathbb{F}_{\mathfrak{p}}\right)\right)$ surjects onto $(A / \mathfrak{p})^{\times} / \mathbb{F}_{q}^{\times}$. Therefore, $\left(M^{1}(\mathfrak{p}) \otimes \mathbb{F}_{T}\right) / \operatorname{det}\left(Z\left(\mathbb{F}_{\mathfrak{p}}\right)\right)=\mathbb{F}_{T} \cong \mathbb{F}_{q}$. The claim of the proposition follows.

Let $K$ be any $A$-field, and $\varphi$ be a rank- $n$ Drinfeld module over $K$. Let $L$ be a field extension of $K$. Denote by $\operatorname{End}_{L}(\varphi)$ the centralizer of $A \stackrel{\cong}{\longrightarrow} \varphi(A)$ in $L\{\tau\}$. More concretely, $\operatorname{End}_{L}(\varphi)$ consists of all $u \in L\{\tau\}$ such that $u \cdot \varphi_{a}=\varphi_{a} \cdot u$ for all $a \in A$. Let $\operatorname{Aut}_{L}(\varphi):=\operatorname{End}_{L}(\varphi)^{\times}$. If $\varphi$ has rank $n$ then, as is easy to check, $\operatorname{Aut}_{L}(\varphi)$ is a subgroup $\mathbb{F}_{q^{s}}^{\times}$of $\mathbb{F}_{q^{n}}^{\times}$, for some $s$ dividing $n$. We denote $\operatorname{End}_{\bar{K}}(\varphi)$ by $\operatorname{End}(\varphi)$, and similarly for $\operatorname{Aut}_{\bar{K}}(\varphi)$. It is known that $\operatorname{End}(\varphi)$ is a free $A$-module of rank less than or equal to $n^{2}$; see $[4, \S 2]$.

Fix some prime $\mathfrak{p}$, and assume $K$ is a finite extension of $\mathbb{F}_{\mathfrak{p}}$. Then $K$ has cardinality $q^{m}$ for some $m$. Let $\operatorname{Fr}_{\mathfrak{p}}=\tau^{m}: x \mapsto x^{q^{m}}$ be the associated (arithmetic) Frobenius morphism. It is clear that $\operatorname{Fr}_{\mathfrak{p}} \in \operatorname{End}_{K}(\varphi)$.

Proposition 4.8. The following conditions are equivalent:

(1) $\left[\operatorname{End}(\varphi) \otimes_{A} F: F\right]=n^{2}$;

(2) $\operatorname{End}(\varphi)$ is a maximal order in the central division algebra over $F$ of dimension $n^{2}$, which is ramified exactly at $\mathfrak{p}$ and $\infty$ with invariants $1 / n$ and $-1 / n$, respectively;

(3) Some power of $\operatorname{Fr}_{\mathfrak{p}}$ lies in $\varphi(A)$;

(4) $\operatorname{ker}\left(\varphi_{\mathfrak{p}}\right)$ is connected.

Proof. See [9, §4] and [5].

Drinfeld modules that satisfy the conditions of the proposition are called supersingular. There are only finitely many such modules in characteristic $\mathfrak{p}$.

Example 4.9. Consider the Drinfeld module $\widetilde{\varphi}$ over $\mathbb{F}_{T}$, given by

$$
\widetilde{\varphi}_{T}=\tau^{n} \text {. }
$$

Since $\widetilde{\varphi}_{T}$ is purely inseparable, $\widetilde{\varphi}$ is super-singular of rank $n$. In fact, this is the only super-singular module in characteristic $T$ (up to an isomorphism), as follows from [8, Thm. 1]. In this case, $\operatorname{Fr}_{T}=\tau$ and $\operatorname{Fr}_{T}^{n} \in \widetilde{\varphi}(A)$. It is easy to see that $\operatorname{Aut}(\widetilde{\varphi}) \cong \mathbb{F}_{q^{n}}^{\times}$. Hence $\mathbb{F}_{q^{n}}\{\tau\} \subset \operatorname{End}(\widetilde{\varphi})$. Since $\mathbb{F}_{q^{n}}\{\tau\}$ is of rank $n^{2}$ over $A$, we conclude from Proposition 4.8 that

$$
\operatorname{End}(\widetilde{\varphi})=\mathbb{F}_{q^{n}}\{\tau\}
$$

It is clear that the center of $\operatorname{End}(\widetilde{\varphi})$ is $\mathbb{F}_{q}\left\{\tau^{n}\right\}$ - the submodule generated by $A$. 
Proposition 4.10. Let $\mathfrak{p}$ be an admissible prime. Then

$$
\# X_{\mathfrak{p}}\left(\mathbb{F}_{q^{n}}\right) \geq \frac{[\Gamma: \Gamma(\mathfrak{p})]}{q^{n}-1} .
$$

Proof. The proof is a modification of the proof of Proposition II.2.19 in [19]. Consider the finite flat covering $\pi: M_{T}^{n}(\mathfrak{p}) \rightarrow M_{T}^{n}(1)$. Generically, its degree is $\# G\left(\mathbb{F}_{\mathfrak{p}}\right)$; cf. [18, Lem. 1.4.2]. This induces a covering

$$
\pi^{\prime}: X_{\mathfrak{p}} \rightarrow M_{T}^{n}(1)
$$

The degree of $\pi^{\prime}$, generically, is $\# \mathrm{PGL}_{n}\left(\mathbb{F}_{\mathfrak{p}}\right)$. Let $\widetilde{\varphi}$ be the Drinfeld module of Example 4.9. The points corresponding to $\widetilde{\varphi}$ are branch points for $\pi^{\prime}$ with indices $\operatorname{Aut}(\widetilde{\varphi}) / \mathbb{F}_{q}^{\times}$(a generic Drinfeld module in any characteristic has automorphism group isomorphic to $\mathbb{F}_{q}^{\times}$). Hence the number of such points on $X_{\mathfrak{p}}$ is equal to $\# \mathrm{PGL}_{n}\left(\mathbb{F}_{\mathfrak{p}}\right)(q-1) /\left(q^{n}-1\right)$. Observe that

$$
\frac{\# \mathrm{PGL}_{n}\left(\mathbb{F}_{\mathfrak{p}}\right)(q-1)}{\left(q^{n}-1\right)}=\frac{[\Gamma: \Gamma(\mathfrak{p})]}{\left(q^{n}-1\right)}
$$

so it suffices to show that all points on $X_{\mathfrak{p}}$ corresponding to $\widetilde{\varphi}$ are rational over $\mathbb{F}_{q^{n}}$. For this, in turn, it suffices to show that any structure $\lambda$ of level $\mathfrak{p}$ on the module $\widetilde{\varphi}$ under the action of $\tau^{n}$ gives a structure lying over the same point in $X_{\mathfrak{p}}$ as the original structure (here the pair $(\widetilde{\varphi}, \lambda)$ is considered as a point of $M_{T}^{n}(\mathfrak{p})$ ).

The action of $\tau^{n}$ on $\lambda$ is via its image under the composition

$$
\operatorname{End}(\widetilde{\varphi}) \rightarrow\left(\operatorname{End}(\widetilde{\varphi}) \otimes_{A_{\mathfrak{p}}} \mathbb{F}_{\mathfrak{p}}\right)^{\times} \stackrel{\sim}{\longrightarrow} G\left(\mathbb{F}_{\mathfrak{p}}\right)
$$

where the last isomorphism follows from Proposition 4.8. Since $\tau^{n}$ lies in the center of $\operatorname{End}(\widetilde{\varphi})$, its image in $G\left(\mathbb{F}_{\mathfrak{p}}\right)$ lies in $Z\left(\mathbb{F}_{\mathfrak{p}}\right)$. This implies that $(\widetilde{\varphi}, \lambda)$ and $\tau^{n}(\widetilde{\varphi}, \lambda)$ have the same image in $X_{\mathfrak{p}}$, so this point is $\mathbb{F}_{q^{n}}$-rational.

4.2. Asymptotic bounds. Recall from the introduction the $\ell$-adic cohomology groups with compact supports

$$
H_{\eta}^{*}(\mathfrak{n}):=H_{c}^{*}\left(M_{\eta}^{n}(\mathfrak{n}) \otimes_{F} \bar{F}, \overline{\mathbb{Q}}_{\ell}\right),
$$

where $M_{\eta}^{n}(\mathfrak{n}):=M^{n}(\mathfrak{n}) \otimes_{A\left[\mathfrak{n}^{-1}\right]} F$. Similarly, for a proper prime ideal $\mathfrak{p}$ of $A\left[\mathfrak{n}^{-1}\right]$, denote $M_{\mathfrak{p}}^{n}(\mathfrak{n}):=M^{n}(\mathfrak{n}) \otimes_{A[\mathfrak{n}-1]} \mathbb{F}_{\mathfrak{p}}$ and $H_{\mathfrak{p}}^{*}(\mathfrak{n}):=H_{c}^{*}\left(M_{\mathfrak{p}}^{n}(\mathfrak{n}) \otimes_{\mathbb{F}_{\mathfrak{p}}} \overline{\mathbb{F}}_{\mathfrak{p}}, \overline{\mathbb{Q}}_{\ell}\right)$.

$H_{\eta}^{*}(\mathfrak{n})$ is endowed with commuting actions of the Galois group $\operatorname{Gal}(\bar{F} / F)$ and a certain Hecke algebra $\mathbb{T}_{\mathfrak{n}}$. Since $M_{\eta}^{n}(\mathfrak{n})$ is a smooth affine scheme of pure relative dimension $(n-1)$ over $F$, the cohomology groups $H_{\eta}^{i}(\mathfrak{n})$ are finite dimensional and vanish for $i \notin[n-1,2(n-1)]$, cf. [18, $\S 12.2]$. Denote by $h_{\eta}^{i}(\mathfrak{n})=\operatorname{dim}_{\overline{\mathbb{Q}}_{\ell}} H_{\eta}^{i}(\mathfrak{n})$, $i \geq 0$, the $\ell$-adic Betti numbers of $M_{\eta}^{n}(\mathfrak{n}) \otimes_{F} \bar{F}$. Similarly, denote by $h_{\mathfrak{p}}^{i}(\mathfrak{n})$ the $\ell$-adic Betti numbers of $M_{\mathfrak{p}}^{n}(\mathfrak{n}) \otimes_{\mathbb{F}_{\mathfrak{p}}} \overline{\mathbb{F}}_{\mathfrak{p}}$.

Proposition 4.11. Fix a proper prime ideal $\mathfrak{p} \triangleleft A$. Under the assumption (1.4), we have

$$
\lim _{\substack{\operatorname{deg}(\mathfrak{n}) \rightarrow \infty \\(\mathfrak{n}, \mathfrak{p})=1}}\left(\frac{\sum_{i \geq 0} h_{\mathfrak{p}}^{i}(\mathfrak{n})}{\operatorname{dim}_{\mathbb{C}} W_{\mathrm{sp}}(\mathfrak{n})}\right)=n,
$$

where the limit is over all ideals $\mathfrak{n} \triangleleft A$ which are coprime to $\mathfrak{p}$. 
Proof. Denote by $W_{\mathrm{sp}}^{m}(\mathfrak{n})$ the space of cusp forms on $\mathrm{GL}_{m}\left(F_{\infty}\right)$ of level $\mathfrak{n}$ which are special at $\infty$ (in particular, $W_{\mathrm{sp}}^{n}(\mathfrak{n})=W_{\mathrm{sp}}(\mathfrak{n})$ in our earlier notation). We claim that for any $\left(p_{1}, \ldots, p_{h}\right) \neq(n) \in \mathbf{P}(n)$

$$
\lim _{\operatorname{deg}(\mathfrak{n}) \rightarrow \infty} \frac{\operatorname{dim}_{\mathbb{C}}\left(W_{\mathrm{sp}}^{p_{1}}(\mathfrak{n}) \otimes \cdots \otimes W_{\mathrm{sp}}^{p_{h}}(\mathfrak{n})\right)}{\operatorname{dim}_{\mathbb{C}} W_{\mathrm{sp}}^{n}(\mathfrak{n})}=0 .
$$

Indeed, using Corollary 3.27, one checks that $\operatorname{dim}_{\mathbb{C}}\left(W_{\mathrm{sp}}^{m}(\mathfrak{n})\right)$ is $O\left(q^{m^{2} \operatorname{deg}(\mathfrak{n})}\right)$ for $\operatorname{deg}(\mathfrak{n}) \gg 0$. On the other hand, $p_{1}^{2}+\cdots+p_{h}^{2}<n^{2}$ if $\left(p_{1}, \ldots, p_{h}\right) \neq(n)$.

To proceed further, we appeal to one of the main results in [18]. The central goal of [18] is to describe the virtual $\operatorname{Gal}(\bar{F} / F) \times \mathbb{T}_{\mathfrak{n}}$-module $\mathcal{H}:=\sum_{i \geq 0}(-1)^{i} H_{\eta}^{i}(\mathfrak{n})$. (This is essentially the Langlands conjecture for cuspidal automorphic irreducible representations of $G(\mathbb{A})$ which are special at $\infty$.) Laumon shows [18, Thm. 12.5.1] that $\mathcal{H}$, as a sum of irreducible $\operatorname{Gal}(\bar{F} / F) \times \mathbb{T}_{\mathfrak{n}}$-modules, is equal to a sum of cuspidal representations of $G(\mathbb{A})$ induced from the representations on $W_{\mathrm{sp}}^{p_{1}}(\mathfrak{n}) \otimes \cdots \otimes W_{\mathrm{sp}}^{p_{h}}(\mathfrak{n})$, $\left(p_{1}, \ldots, p_{h}\right) \in \mathbf{P}(n)$, tensored with the Galois representations attached to these cuspidal representations by the Langlands correspondence (the same theorem for $n=2$ is due to Drinfeld [4]). If we assume (1.4), then Laumon's theorem and (4.1) imply

$$
\lim _{\operatorname{deg}(\mathfrak{n}) \rightarrow \infty}\left(\frac{\sum_{i \geq 0} h_{\eta}^{i}(\mathfrak{n})}{\operatorname{dim}_{\mathbb{C}} W_{\mathrm{sp}}^{n}(\mathfrak{n})}\right)=n .
$$

Now, for any $i$ the $\operatorname{Gal}(\bar{F} / F)$-module $H_{\eta}^{i}$ is unramified away from $\operatorname{supp}(\mathfrak{n}) \cup\{\infty\}$, and moreover, for each proper ideal $\mathfrak{p}$ of $A\left[\mathfrak{n}^{-1}\right]$ there is a $\operatorname{Gal}\left(\overline{\mathbb{F}}_{\mathfrak{p}} / \mathbb{F}_{\mathfrak{p}}\right)$-equivariant isomorphism $H_{\eta}^{i} \cong H_{\mathfrak{p}}^{i}$. Besides some theorems from SGA, the proof of this fact uses Pink's construction of toroidal compactifications of $M^{n}(\mathfrak{n})$; see (12.2.2.1), (12.2.2.2) and (12.2.7) in [18]. We conclude that $\sum_{i \geq 0} h_{\eta}^{i}(\mathfrak{n})=\sum_{i \geq 0} h_{\mathfrak{p}}^{i}(\mathfrak{n})$, and the theorem follows.

Remark 4.12. The statement (1.4) would follow if one could understand not just $\mathcal{H}$ as a $\operatorname{Gal}(\bar{F} / F) \times \mathbb{T}_{\mathfrak{n}}$-module, but the individual cohomology groups $H_{\eta}^{i}(\mathfrak{n})$ as such modules. For example, it is likely that the Galois representations which correspond to $W_{\mathrm{sp}}^{n}(\mathfrak{n})$ occur only in $H_{\eta}^{n-1}(\mathfrak{n})$ and $h_{\eta}^{n-1}(\mathfrak{n}) \sim n \operatorname{dim}_{\mathbb{C}} W_{\mathrm{sp}}^{n}(\mathfrak{n})$.

Let $\operatorname{Frob}_{\mathfrak{p}}=\operatorname{Fr}_{\mathfrak{p}}^{-1} \in \operatorname{Gal}\left(\overline{\mathbb{F}}_{\mathfrak{p}} / \mathbb{F}_{\mathfrak{p}}\right)$ be the geometric Frobenius element. If $\mathfrak{p} \notin$ $\operatorname{supp}(\mathfrak{n})$ then Frob $_{\mathfrak{p}}$ defines an automorphism of $H_{c, \mathfrak{p}}^{i}(\mathfrak{n})$. Assume $H_{c, \mathfrak{p}}^{i}(\mathfrak{n}) \neq 0$. Denote the eigenvalues of Frob $\mathrm{p}_{\mathfrak{p}}$ acting on this finite dimensional $\overline{\mathbb{Q}}_{\ell}$-vector space by $\alpha_{i, 1}, \alpha_{i, 2}, \ldots, \alpha_{i, s}\left(\right.$ here $\left.s=h_{\mathfrak{p}}^{i}(\mathfrak{n})\right)$.

Proposition 4.13. Fix a proper prime ideal $\mathfrak{p} \triangleleft A$ and $k \in \mathbb{Z}_{\geq 0}$. Under the assumption (1.4), we have

$$
\lim _{\substack{\operatorname{deg}(\mathfrak{n}) \rightarrow \infty \\(\mathfrak{n}, \mathfrak{p})=1}}\left(\frac{\sum_{i \geq 0} \sum_{j=1}^{h_{\mathfrak{p}}^{i}(\mathfrak{n})}\left|\alpha_{i, j}^{k}\right|}{\sum_{i \geq 0} h_{\mathfrak{p}}^{i}(\mathfrak{n})}\right)=q^{\operatorname{deg}(\mathfrak{p}) k \frac{(n-1)}{2}} .
$$

Proof. Let $\sum_{\mathrm{sp}}\left|\alpha_{i, j}^{k}\right|$ be the sum over the eigenvalues $\alpha_{i, j}$ corresponding to $W_{\mathrm{sp}}^{n}(\mathfrak{n})$ under the Langlands conjecture; see Theorem 12.4.1, Theorem 12.5.1, and Corollary 12.4.9 in [18]. By the Ramanujan-Petersson conjecture [18, Thm.12.4.1]

$$
\sum_{\mathrm{sp}}\left|\alpha_{i, j}^{k}\right|=q^{\operatorname{deg}(\mathfrak{p}) k \frac{(n-1)}{2}} n \operatorname{dim}_{\mathbb{C}}\left(W_{\mathrm{sp}}(\mathfrak{n})\right) .
$$


On the other hand, by Deligne's theorem [3, Thm. 3.3.1] all $\left|\alpha_{i, j}^{k}\right|$ are bounded by $q^{\operatorname{deg}(\mathfrak{p}) k(n-1)}$, which is independent of $\mathfrak{n}$. Hence, our claim follows from Proposition 4.11 .

Theorem 4.14. Let $\mathfrak{p} \neq T$ be an admissible prime. Let $X_{\mathfrak{p}}$ be the smooth, geometrically irreducible affine variety over $\mathbb{F}_{q}$ which we constructed in $\S 4.1$.

$$
\begin{gathered}
\frac{1}{n} \prod_{j=1}^{n-1}\left(q^{j}-1\right) \leq \liminf _{\operatorname{deg}(\mathfrak{p}) \rightarrow \infty}\left(\frac{\# X_{\mathfrak{p}}\left(\mathbb{F}_{q^{n}}\right)}{\sum_{i \geq 0} h^{i}\left(X_{\mathfrak{p}}\right)}\right), \\
\quad \limsup _{\operatorname{deg}(\mathfrak{p}) \rightarrow \infty}\left(\frac{\# X_{\mathfrak{p}}\left(\mathbb{F}_{q^{n}}\right)}{\sum_{i \geq 0} h^{i}\left(X_{\mathfrak{p}}\right)}\right) \leq q^{\frac{n(n-1)}{2}} .
\end{gathered}
$$

Proof. $M_{T}^{n}(\mathfrak{p}) \otimes_{\mathbb{F}_{q}} \overline{\mathbb{F}}_{q}$ is a disjoint union of $\left[(A / \mathfrak{p})^{\times}: \mathbb{F}_{q}^{\times}\right]$copies of $X_{\mathfrak{p}} \otimes_{\mathbb{F}_{q}} \overline{\mathbb{F}}_{q}$. Hence

$$
\sum_{i \geq 0} h_{T}^{i}(\mathfrak{n})=\left[(A / \mathfrak{p})^{\times}: \mathbb{F}_{q}^{\times}\right] \sum_{i \geq 0} h^{i}\left(X_{\mathfrak{p}}\right) .
$$

This equality, combined with Corollary 3.27 and Proposition 4.11, implies

$$
\lim _{\operatorname{deg}(\mathfrak{p}) \rightarrow \infty}\left(\frac{\sum_{i \geq 0} h^{i}\left(X_{\mathfrak{p}}\right)}{[\Gamma: \Gamma(\mathfrak{p})]}\right)=\frac{n}{\left(q^{n}-1\right) \cdots(q-1)} .
$$

Now the lower bound in the theorem follows from Proposition 4.10.

Next, recall that by the Grothendieck-Lefschetz trace formula

$$
\begin{aligned}
\# X_{\mathfrak{p}}\left(\mathbb{F}_{q^{n}}\right) & =\sum_{i \geq 0}(-1)^{i} \operatorname{Tr}\left(\operatorname{Frob}_{T}^{n} \mid H_{c}^{i}\left(X_{\mathfrak{p}} \otimes_{\mathbb{F}_{q}} \overline{\mathbb{F}}_{q}, \overline{\mathbb{Q}}_{\ell}\right)\right) \\
& =\frac{1}{\left[(A / \mathfrak{p})^{\times}: \mathbb{F}_{q}^{\times}\right]} \sum_{i \geq 0}(-1)^{i} \sum_{j=1}^{h_{T}^{i}(\mathfrak{p})} \alpha_{i, j}^{n} .
\end{aligned}
$$

Hence

$$
\# X_{\mathfrak{p}}\left(\mathbb{F}_{q^{n}}\right) \leq \frac{1}{\left[(A / \mathfrak{p})^{\times}: \mathbb{F}_{q}^{\times}\right]} \sum_{i \geq 0} \sum_{j=1}^{h_{T}^{i}(\mathfrak{p})}\left|\alpha_{i, j}^{n}\right|
$$

and the upper bound in the theorem follows from Proposition 4.13 and (4.2).

\section{REFERENCES}

1. G. Andrews, The theory of partitions, Cambridge Univ. Press, 1984.

2. W. Casselman, On a p-adic vanishing theorem of Garland, Bull. Amer. Math. Soc. 80 (1974), 1001-1004.

3. P. Deligne, La conjecture de Weil: II, Publ. Math. IHÉS 52 (1980), 137-252.

4. V. Drinfeld, Elliptic modules, Math. Sbornik 94 (1974), 594-627.

5. __ Elliptic modules: II, Math. Sbornik 102 (1977), 182-194.

6. H. Garland, p-adic curvature and the cohomology of discrete groups, Ann. Math. 97 (1973), $375-423$.

7. M. Gebhardt, Operationen arithmetischer Untergruppen von GL(3) auf Bruhat-TitsGebäuden, Diplomarbeit, University of Saarland (1996).

8. E.-U. Gekeler, Sur les classes d'idéaux des ordres de certains corps gauches, C. R. Acad. Sci. Paris Sér. I Math. 309 (1989), 577-580.

9. _ , On finite Drinfeld modules, J. Algebra 141 (1991), 187-203.

10. - Invariants of some algebraic curves related to Drinfeld modular curves, J. of Number Theory 90 (2001), 166-183. 
11. E.-U. Gekeler and U. Nonnengardt, Fundamental domains of some arithmetic groups over function fields, Internat. J. Math. 6 (1995), 689-708.

12. G. Harder, Minkowskische Reduktionstheorie über Funktionenkörpern, Invent. Math. 7 (1969), $33-54$.

13. Chevalley groups over function fields and automorphic forms, Ann. Math. 100 (1974), 249-306.

14. _ Die Kohomologie S-arithmetischer Gruppen über Funktionenkörpern, Invent. Math. 42 (1977), 135-175.

15. R. Hartshorne, Algebraic geometry, Springer, 1977.

16. P. Hatcher, Algebraic topology, Cambridge Univ. Press, 2002.

17. D. Hayes, Explicit class field theory for rational function fields, Tran. Amer. Math. Soc. 189 (1974), 77-91.

18. G. Laumon, Cohomology of Drinfeld modular varieties: I, II, Cambridge Univ. Press, 1996/1997.

19. Yu. Manin and S. Vladut, Linear codes and modular curves, J. Sov. Math. 30 (1985), 26112643.

20. J. Milne, Étale cohomology, Princeton Univ. Press, 1980.

21. J.-P. Serre, Cohomologie des groupes discrets, Ann. Math. Studies 70 (1970), 77-169.

22. _ Trees, Springer Monographs in Math., 2003.

23. C. Soulé, Chevalley groups over polynomial rings, LMS Lect. Notes 36 (1979), 359-367.

24. G.-J. van der Heiden, Drinfeld modular curves and Weil pairing, arXiv: math.AG/0411490 (2004).

25. Weil pairing for Drinfeld modules, Monatsh. Math. 143 (2004), 115-143.

26. S. Vladut and V. Drinfeld, The number of points of an algebraic curve, Funct. Anal. Appl. 17 (1983), 53-54

Department of Mathematics, Stanford University, Stanford, CA 94305, U.S.A.

E-mail address: papikian@math.stanford.edu 